\title{
SENSITIVITY OF ADOP TO CHANGES IN THE SINGLE-BASELINE GNSS MODEL
}

\author{
D. Odijk, P.J.G. Teunissen \\ Delft Institute of Earth Observation and Space Systems (DEOS) \\ Delft University of Technology \\ Kluyverweg 1, 2629 HS Delft, The Netherlands \\ e-mail: d.odijk@tudelft.nl, p.j.g.teunissen@tudelft.nl
}

\begin{abstract}
The ADOP (Ambiguity Dilution Of Precision) is a measure for the precision of the carrier phase ambiguities involved in precise relative GNSS positioning. By computing the ADOP one may get knowledge in whether ambiguity resolution can be expected successful or not, already in a stage before the GNSS data are collected. In Odijk and Teunissen (2008) compact closed-form expressions have been derived for the ADOP of single-baseline GNSS models. In this paper these expressions are used to study the impact of certain changes in these models, as there are the observation time span, the weighting of the ionospheric delays, the number of frequencies, the weights of the phase and code data, the number of satellites, elevation-dependent observation weights and taking linear combinations of data.
\end{abstract}

Keywords: GNSS models, ambiguity resolution, ADOP.

\section{INTRODUCTION}

The Ambiguity Dilution Of Precision (ADOP) is a simple scalar measure to assess the precision of the carrier-phase ambiguities. A high precision is crucial to the success of the resolution of the integer ambiguities, a process which is the key to fast precise relative GNSS positioning. In Odijk and Teunissen (2008) it was shown that analytical closedform expressions could be derived for a whole range of single-baseline GNSS models for precise relative positioning. In this contribution these closed-form expressions are used to analyze the sensitivity of the ADOP to changes in these models.

Single-baseline GNSS models can be divided into geometry-free (GFr) models, in which the relative receiver-satellite geometry is dispensed, geometry-based (GB) models, the usual model for positioning and hence taking the receiver-satellite geometry into account, and geometry-fixed models, in which the positions of both receivers of the baseline are held fixed to their known values. The geometry-free and geometry-fixed model are included here since they serve as a beneficial tool for the derivation of the closed-form expressions of the geometry-based models. Within the class of geometry-based models a further distinction can be made, namely between models that comprise only short observation 
time spans and those comprising long time spans. For short-time models one may with a fairly good approximation- assume that the receiver-satellite geometry is constant in time, which facilitates the derivation of closed-form expressions for the ADOP. In case of the short-time model we distinguish between models in which both receivers are stationary (the stationary-receiver short-time, or briefly SR-ST, model) and models in which one of the receivers is moving (the moving-receiver short-time, or briefly MRST model). In case of a long observation time span we only assume receivers that are stationary and the model is referred to as the stationary-receiver long-time, or briefly SRLT, model. Figure 1 depicts schematically the GNSS models considered in this paper. For all models it holds that a priori ionospheric information in the form of ionospheric pseudo observables can be added to the multi-frequency phase and code observables. In this way the behavior of the ionospheric delays is tuned or weighted in the models, see Teunissen (1998). Hence we speak of ionosphere-weighted models. In our considered models, all observables and parameters are double-differenced (DD) relative to a reference receiver and reference satellite.

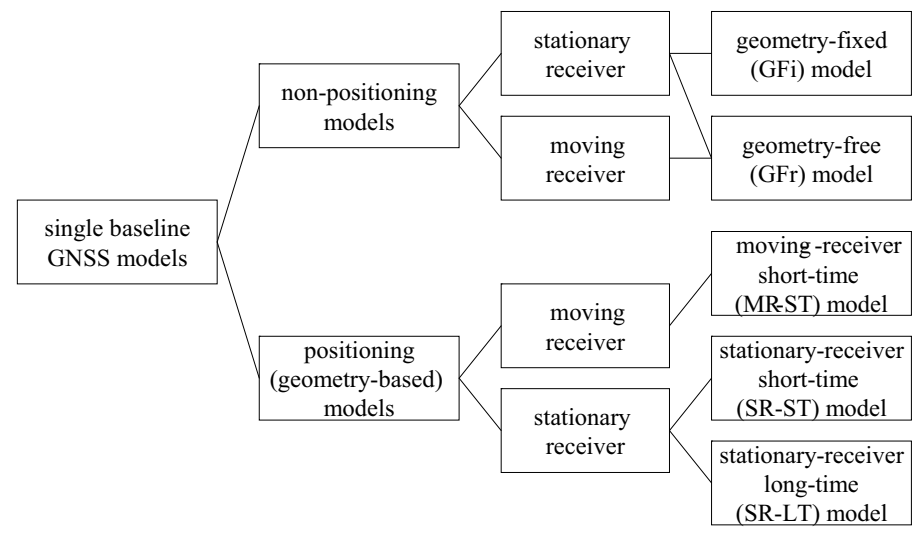

Figure 1: Considered versions of the GNSS single-baseline model.

This paper is set up as follows. Section 2 reviews the closed-form ADOP expressions for the single-baseline model. In Section 3 the ADOPs of the different types of singlebaseline models are compared. Section 4 presents an analysis of the sensitivity of changes in the model assumptions on the size of the ADOP. The paper ends with concluding remarks and a summarizing table. Although all tables and examples in this paper focus on GPS, the derivations are valid for the whole range of GNSS.

\section{ADOP AS A PRODUCT OF FIVE SCALARS}

The ADOP measure is defined as $A D O P=\left|Q_{\hat{a}}\right|^{1 /(2 n)}$, with $|\cdot|$ the determinant operator, $Q_{\hat{a}}$ the variance-covariance (vc) matrix of the (float) DD ambiguities, and $n$ the number of DD ambiguities involved (Teunissen, 1997a). In Odijk and Teunissen (2008) it was shown that the closed-form ADOP expressions for the single-baseline models in Fig. 1 can be written as a product of five scalars:

$$
A D O P=f_{1} \times f_{2} \times f_{3} \times f_{4} \times f_{5} \quad[\mathrm{cyc}]
$$

In this section we will review how each of these five scalars are computed for the GFr, MR-ST, SR-ST, SR-LT and GFi models. For all models it is assumed that GNSS phase 
and code data of $m$ satellites are (simultaneously) tracked by two $j$-frequency receivers with a constant sampling interval during $k$ observation epochs.

The first factor, $f_{1}$, is computed as:

$$
f_{1}= \begin{cases}m^{\frac{1}{2(m-1)}} & \text { GFr, SR-LT, GFi } \\ {\left[\frac{\sum_{s=1}^{m} w_{s}}{\prod_{s=1}^{m} w_{s}}\right]^{\frac{1}{2(m-1)}}} & \text { MR-ST, SR-ST }\end{cases}
$$

As can be seen, in this factor the number of satellites $m$ plays a crucial role. In case of the two short-time geometry-based models one may account for satellite-dependent weighting through the satellite weights $w_{s}$, for $s=1, \ldots, m$. In absence of this satellite-dependent weighting, these weights are set equal to 1 implying that factor $f_{1}$ becomes equal for all types of models considered. Note that satellite-dependent weighting is not taken into account for the geometry-free model, since this model only serves as auxiliary tool to facilitate the understanding of the other models. It is not taken into account for the SR-LT model as well, since the effect of satellite-dependent weighting will more or less be averaged out because of the long observation time.

The second factor, $f_{2}$ is computed as:

$$
f_{2}= \begin{cases}\sqrt{\frac{2}{k}} \quad \text { GFr, SR-LT, GFi } \\ \sqrt{\frac{2}{e_{k}^{T} R_{k}^{-1} e_{k}}} \text { MR-ST, SR-ST }\end{cases}
$$

In this factor the number of epochs $k$ comprising the observation time span plays a crucial role. For the two short-time geometry-based models it is through matrix $R_{k}$ (of dimension $k$ ) possible to account for time correlation between epochs. For the geometry-free model time correlation is not considered for the same reason as satellite-dependent weighting, while for the long-time geometry-based model time correlation can be easily omitted by increasing the sampling interval of the observations. Later on in this paper we will focus on the structure of time correlation matrix $R_{k}$. In absence of time correlation, this matrix equals the $k$-dimensional identity matrix and with $e_{k}$ defined as the $k$-dimensional vector having 1 at all entries, the scalar $e_{k}^{T} R_{k}^{-1} e_{k}$ simply reduces to $k$.

The third factor, $f_{3}$, is equal for all types of models and is computed as:

$$
f_{3}=\frac{\mid C_{\phi} \frac{1}{2 j}}{\grave{\lambda}}, \quad \text { where } \breve{\lambda}=\prod_{i=1}^{j} \lambda_{i}^{1 / j}
$$

In this expression $j$ denotes the number of frequencies on which the GNSS data are collected, whereas $\left|C_{\phi}\right|$ denotes the determinant of the $j$-dimensional vc-matrix of the undifferenced phase observables. Scalar $\breve{\lambda}$ denotes the geometric mean of the wavelengths corresponding to the $j$ frequencies. Note that in absence of correlation between the phase observables, $C_{\phi}$ reduces to the $j$-dimensional identity matrix and the numerator of $f_{3}$ reduces to the geometric mean of the standard deviations of the $j$ undifferenced phase observables, i.e. $\left|C_{\phi}\right|^{\frac{1}{2 j}}=\prod_{i=1}^{j} c_{\phi_{i}}^{1 / j}$.

Like the third factor also the fourth factor, $f_{4}$, is equivalent for all types of models considered. It is computed as:

$$
f_{4}=\left[1+\frac{1}{\iota}\right]^{\frac{1}{2 j}}, \quad \text { where } \iota=\frac{1}{c_{i \mid \rho}^{2} / c_{i \mid \rho}^{2}-1}
$$


Scalar $\iota$ is a function of the ratio of the ambiguity-float and -fixed ionospheric variance factors that are conditioned on the ranges, i.e. $c_{\hat{\imath} \mid \rho}^{2}$ and $c_{\hat{\imath} \mid \rho}^{2}$, estimated using the geometryfree model. This ratio is the following function of the a priori precision of the undifferenced phase, code and ionosphere observables:

$$
\frac{c_{i \mid \rho}^{2}}{c_{\tilde{\imath} \mid \rho}^{2}}=\frac{\left[\mu^{T}\left(C_{\phi}^{-1}+C_{p}^{-1}\right) \mu\right]+c_{\imath}^{-2}}{\left[\mu^{T} C_{p}^{-1} \mu\right]+c_{\imath}^{-2}}
$$

Here $j \times j$-matrices $C_{\phi}$ and $C_{p}$ denote the vc-matrices of the undifferenced phase and code data, respectively, whereas the $j$-vector $\mu$ contains the ratios of squared wavelengths with the wavelength of the first frequency: $\mu=\left(\mu_{1}, \ldots, \mu_{j}\right)^{T}$, with $\mu_{j}=\lambda_{j}^{2} / \lambda_{1}^{2}$. Scalar $c_{\imath}^{2}$ denotes the variance of the undifferenced ionospheric pseudo observables. Factor $f_{4}$ is thus strongly governed by the weight (the reciprocal value of the variance) of the ionospheric pseudo observables in the model. When $c_{\imath}^{-2}=\infty$, this ionosphere-weighted model is referred to as the ionosphere-fixed model and when $c_{\imath}^{-2}=0$, it is referred to as the ionosphere-float model.

The fifth factor, $f_{5}$, is different for the types of single-baseline models considered:

$$
f_{5}=\left\{\begin{array}{lr|}
{\left[1+\frac{1}{\delta}\right]^{\frac{1}{2 j}}} & \text { GFr } \\
{\left[1+\frac{1}{\delta}\right]^{\frac{v}{2 j(m-1)}}} & \text { MR-ST, } \\
{\left[\prod_{i=1}^{v}\left(1+\frac{1-1 / \gamma_{i}}{\delta+1 / \gamma_{i}}\right)\right]^{\frac{1}{2 j(m-1)}}} & \text { SR-LT } \\
1 & \text { GFi }
\end{array}\right.
$$

In Eq. (7), scalar $\delta$ is computed as $\delta=\frac{1}{c_{\hat{\rho}}^{2} / c_{\hat{\rho}}^{2}-1}$, where $c_{\hat{\rho}}^{2} / c_{\tilde{\rho}}^{2}$ is the ratio of ambiguity-float and -fixed range variance factors, estimated using the geometry-free model. This ratio is computed as:

$$
\frac{c_{\rho}^{2}}{c_{\tilde{\rho}}^{2}}=\frac{\left[\mu^{T} C_{p}^{-1} \mu\right]+c_{\imath}^{-2}}{\left[e_{j}^{T} C_{p}^{-1} e_{j}\right]\left(\left[\mu^{T} C_{p}^{-1} \mu\right]+c_{\imath}^{-2}\right)-\left[e_{j}^{T} C_{p}^{-1} \mu\right]^{2}} \cdot \frac{\left[e_{j}^{T}\left(C_{\phi}^{-1}+C_{p}^{-1}\right) e_{j}\right]\left(\left[\mu^{T}\left(C_{\phi}^{-1}+C_{p}^{-1}\right) \mu\right]+c_{\imath}^{-2}\right)-\left[e_{j}^{T}\left(C_{\phi}^{-1}-C_{p}^{-1}\right) \mu\right]^{2}}{\left[\mu^{T}\left(C_{\phi}^{-1}+C_{p}^{-1}\right) \mu\right]+c_{\imath}^{-2}}
$$

Like the scalar $\iota, \delta$ is a function of the a priori precision of the undifferenced phase, code and ionosphere observables. This fifth factor accounts for the most essential difference between the models considered: the inclusion of the receiver-satellite geometry or not. In the geometry-free model the receiver-satellite geometry is not included, since for every epoch (DD) receiver-satellite ranges are estimated. In both short-time models (movingand stationary receiver) the receiver-satellite geometry is taken into account, though in the time-constant approximation, since for short time spans the receiver-satellite constellation does not change significantly (due to the high altitude of the GNSS satellites). Note from Eq. (7) that the difference between the factors $f_{5}$ of the geometry-free and short-time models lies in the power, in which the scalar $v$ appears in case of the short-time models. This scalar reflects the number of geometry parameters to be estimated, i.e. $v=3$ in case of three coordinate components and $v=4$ in case also a tropospheric (zenith delay) parameter is estimated, aside from the three coordinate components. In case $m=v+1$ the factor $f_{5}$ of the short-time models equals its geometry-free counterpart. In contrast to the short-time models, for the long-time model we must account for the changing geometry in time. In Odijk and Teunissen (2008) it was shown that the baseline gain number concept easily allows for this changing receiver-satellite geometry in the closed-form ADOP expressions. For each baseline component (including the tropospheric 
zenith delay parameter) we can compute a gain number $\gamma_{i}$ that only depends on the receiver-satellite unit direction vectors (or the mapping function coefficients in case of the tropospheric zenith delay) during the observation time span. These gain numbers in fact measure the gain in baseline precision due to ambiguity fixing and they may range from 1 (i.e. no gain; in case of an infinity long time span) to infinity (i.e. maximum gain; in case of an infinitely short time span, i.e. a single epoch).

\section{THE ADOPS OF THE SINGLE-BASELINE MODELS COMPARED}

Using the closed-form expressions it is possible to rank the ADOPs of the different singlebaseline models.

As can be immediately seen from the five factors in the previous section, for the shorttime ADOP it does not matter whether the second receiver is in motion or not, since the factors of both MR-ST and SR-ST models are equivalent. In absence of satellitedependent weighting and time correlation this short-time ADOP become also equal to the ADOP of the geometry-free model, provided that the number of satellites equals $m=v+1$. With more satellites available, the short-time ADOP (denoted using 'ST') can be written as the following function of the geometry-free ADOP (denoted using 'GFr'):

$$
A D O P^{S T}=\left[1+\frac{1}{\delta}\right]^{\frac{v-(m-1)}{2 j(m-1)}} A D O P^{G F r} \text { for } m \geq v+1
$$

Since the phase precision is better than the code precision, it easily follows using Eq. (8) that $1+\frac{1}{\delta}>1$. Since $m \geq v+1$, the power in the multiplication factor in Eq. (9) is always smaller than or equal to zero. When the power equals zero, the term is exactly 1. For negative powers the term will always be between 0 and 1 , since $1+\frac{1}{\delta}>1$. This proves that $0<\left[1+\frac{1}{\delta}\right]^{\frac{v-(m-1)}{2 j(m-1)}} \leq 1$, and consequently, that the short-time ADOP is smaller than or at most equal to the geometry-free $\mathrm{ADOP}$, i.e. $A D O P^{S T} \leq A D O P^{G F r}$. This is understandable, since the inclusion of the (time-constant) receiver-satellite geometry strengthens the single-baseline model and thus ADOP.

Also, in absence of satellite-dependent weighting and time correlation, the short-time ADOP only differs from its long-time counterpart (SR-LT model) in factor $f_{5}$. They become equal if $\gamma_{i}=\infty$ for $i=1, \ldots, v$ and this is logical, since in case of the short-time models the receiver-satellite geometry does not change, which corresponds to a singleepoch geometry. It can be proved that the long-time ADOP can be written as the following function of the short-time ADOP:

$$
A D O P^{L T}=\left[\prod_{i=1}^{v} \frac{\delta}{\delta+1 / \gamma_{i}}\right]^{\frac{1}{2 j(m-1)}} A D O P^{S T}
$$

Since $\delta \in(0, \infty)$ and $1 / \gamma_{i} \in(0,1]$, for $i=1, \ldots, v$, it follows that $0<\frac{\delta}{\delta+1 / \gamma_{i}} \leq 1$ and thus $0<\left[\prod_{i=1}^{v} \frac{\delta}{\delta+1 / \gamma_{i}}\right] \leq 1$. Since the power $1 / 2 j(m-1)$ is always positive, it follows that the multiplication factor in Eq. (10) is bounded between 0 and 1 and consequently $A D O P^{L T} \leq A D O P^{S T}$. This is also what would be expected, since the changing relative receiver-satellite geometry in time, as included in the long-time model, has a beneficial impact on ADOP.

On the other hand, with $\gamma_{i}=1$ for $i=1, \ldots, v$, the factor $f_{5}$ of the long-time model equals 1 , corresponding to the factor $f_{5}$ of the geometry-fixed model. Since it holds that 
$1+\frac{1-1 / \gamma_{i}}{\delta+1 / \gamma_{i}} \geq 1$ for $i=1, \ldots, v$, it can be proved that $\left[\prod_{i=1}^{v}\left(1+\frac{1-1 / \gamma_{i}}{\delta+1 / \gamma_{i}}\right)\right]^{\frac{1}{2 j(m-1)}} \geq 1$. From this follows that the geometry-fixed ADOP is always smaller or at least equal to the long-time $\mathrm{ADOP}$, i.e. $A D O P^{G F i} \leq A D O P^{L T}$. This is also not surprising, since in the geometry-fixed model there are less parameters than in the SR-LT model. In fact, the ADOPs of both models become equal when the observation time span is infinitely long. Due to the enormous amount of observations in that case, the baseline parameters estimated using the SR-LT model become in fact deterministic or known quantities.

In general, the ADOPs of the different versions of the single-baseline model versions can be arranged as follows:

$$
A D O P^{G F i} \leq A D O P^{L T} \leq A D O P^{S T} \leq A D O P^{G F r} \text { if } m \geq v+1
$$

\section{IMPACT OF CHANGES IN THE SINGLE-BASELINE GNSS MODEL}

In this section we will use the derived closed-form expressions to study the effect on ADOP of changing the single-baseline GNSS model with respect to:

1. observation time span + time correlation

2. ionospheric weighting

3. number of frequencies

4. phase and code weights

5. number of satellites

6. satellite-dependent weighting

7. taking linear combinations of data

This investigation will be carried out by deriving (approximated) expressions for the factors with which the ADOP needs to be multiplied to obtain its value under the changed model. The analytical derivations will be supported by graphical examples, which are based on the receiver-satellite geometry for permanent GPS station Delft $\left(52.0^{\circ} \mathrm{N}, 4.4^{\circ} \mathrm{E}\right)$ in the Netherlands, during the complete day of 1 January 2003 (00-24h UTC; 30s sampling interval; cut-off elevation: $15 \mathrm{deg}$ ). Unless stated otherwise, in all examples it is assumed that both phase and code data are uncorrelated, i.e. $C_{\phi}=c_{\phi}^{2} I_{j}$ and $C_{p}=c_{p}^{2} I_{j}$, with undifferenced standard deviation of $c_{\phi}=3 \mathrm{~mm}$ and $c_{p}=30 \mathrm{~cm}$, respectively. In addition, neither satellite-dependent weighting nor time correlation is assumed unless stated otherwise. In the examples besides the ADOP also ADOP-based ambiguity success rates are computed. These success rates predict the probability of correct integer ambiguity estimation. In Odijk and Teunissen (2008) it is shown that it can be easily computed as:

$$
P_{A D O P}=\left[2 \Phi\left(\frac{1}{2 A D O P}\right)-1\right]^{n}
$$

where $n$ is the number of ambiguities and $\Phi(x)=\int_{-\infty}^{x} \frac{1}{\sqrt{2 \pi}} \exp \left\{-\frac{1}{2} v^{2}\right\} d v$. 


\subsection{Effect of the observation time span}

It is assumed that GNSS data are collected during $k$ epochs with a constant sampling interval $T$, such that the observation time span is $(k-1) T$. This time span can thus be increased through two factors: either increasing the number of samples $k$, or the length between the samples, the interval $T$.

The number of samples $k$ shows up at two places in the closed-form ADOP expressions: in factor $f_{2}$ and -although hidden- in the computation of the baseline gain numbers $\gamma_{i}$ in factor $f_{5}$, in case of the long-time geometry-based model (see Odijk and Teunissen (2008)). The sampling interval $T$ does show up in factor $f_{2}$ of the short-time models in case time correlation is present in the observations. Besides, in case of the long-time model it is -like the number of epochs- hidden in the computation of the baseline gain numbers used in factor $f_{5}$.

\subsubsection{Effect for the short-time geometry-based models}

We will first look at the effect of increasing the time span in case of the short-time models. For this purpose the noise of the observations is considered as a first-order autoregressive stochastic process. In that case, the correlation between an observation at epoch $i$ and an observation at epoch $j$ is described by $\beta^{|i-j|}$, with $0 \leq \beta<1$, see e.g. Priestley (1981). The $k \times k$-correlation matrix $R_{k}$ in factor $f_{2}$ then reads:

$$
R_{k}=\left[\begin{array}{ccccc}
1 & \beta & \ldots & \beta^{k-2} & \beta^{k-1} \\
\beta & 1 & \ldots & \beta^{k-3} & \beta^{k-2} \\
\vdots & \vdots & \ddots & \vdots & \vdots \\
\beta^{k-2} & \beta^{k-3} & \ldots & 1 & \beta \\
\beta^{k-1} & \beta^{k-2} & \ldots & \beta & 1
\end{array}\right], 0 \leq \beta<1
$$

Here we assume that $\beta=\exp \left\{-\frac{T}{\tau}\right\}$, with $T$ the sampling interval of the observations and $\tau$ the correlation time of the noise. Such a process is also referred to as exponentially correlated noise. To evaluate factor $f_{2}$, we need the inverse of the correlation matrix. It is computed as:

$$
R_{k}^{-1}=\frac{1}{1-\beta^{2}}\left[\begin{array}{ccccc}
1 & -\beta & & & \\
-\beta & \left(1+\beta^{2}\right) & -\beta & & \\
& \ddots & \ddots & \ddots & \\
& & -\beta & \left(1+\beta^{2}\right) & -\beta \\
& & & -\beta & 1
\end{array}\right]
$$

Using this, it follows that $\left[e_{k}^{T} R_{k}^{-1} e_{k}\right]=\frac{k-(k-2) \beta}{1+\beta}$. Factor $f_{2}$ can then be rewritten as:

$$
f_{2}=\sqrt{\frac{2}{\left[e_{k}^{T} R_{k}^{-1} e_{k}\right]}}=\sqrt{\frac{2(1+\beta)}{k-(k-2) \beta}}
$$

Note that in absence of time correlation, $\beta=0$, and $f_{2}$ reduces to $\sqrt{2 / k}$. With maximum time correlation, i.e. $\beta=1, f_{2}$ reduces to $\sqrt{2}$.

Suppose that the number of samples is increased with $z$ samples, then the following relation can be derived between the ADOP based on $k$ epochs and the ADOP based on $k+z$ epochs:

$$
A D O P^{S T}(k+z)=\sqrt{\frac{k-(k-2) \beta}{k+z-(k+z-2) \beta}} A D O P^{S T}(k)
$$


where 'ST' stands for short-time model. Since $0 \leq \beta<1$, the scale factor in Eq. (16) interpolates between $\sqrt{\frac{k}{k+z}}$ (for $\beta=0$ ) and 1 (for $\beta=1$ ). For example, in absence of time correlation with $k=1$ and $z=9$, the ADOP corresponding to the time span of 10 epochs is about $\sqrt{1 / 10} \simeq 0.32$ times the single-epoch ADOP. This is illustrated in Fig. 2, in which for a fictitious short baseline (such that the ionosphere-fixed model may be used) the ADOPs, ADOP-based success-rates and number of satellites are plotted during the day. The left graphs concern 2880 single-epoch solutions, while in the right graphs the day is divided into 288 time spans of each 5 minutes. From the graphs can be seen that instantaneous ambiguity resolution based on single-frequency data cannot be expected successful; only with at least 9 satellites the ADOP-based success rate approaches 1. Increasing the time span to 5 minutes improves a lot; only with less than 6 satellites the success rate will be very poor. Note that these 5 minute time spans comprise 10 epochs with 30 sec. sampling interval; if the sampling interval is much shorter, a much shorter time span is needed (in the not realistic case of absence of time correlation). Presence of time correlation of say, $\beta=0.75$ results in an ADOP corresponding to 10 epochs of $\sqrt{1.75 / 4} \simeq 0.66$ times the single-epoch ADOP. This leads to the conclusion that the more temporal correlation between the observations, the less the precision of the ambiguities improves when the number of samples is increased. Note that in the extreme case the observations are fully correlated in time, the precision of the ambiguities is not improved at all.
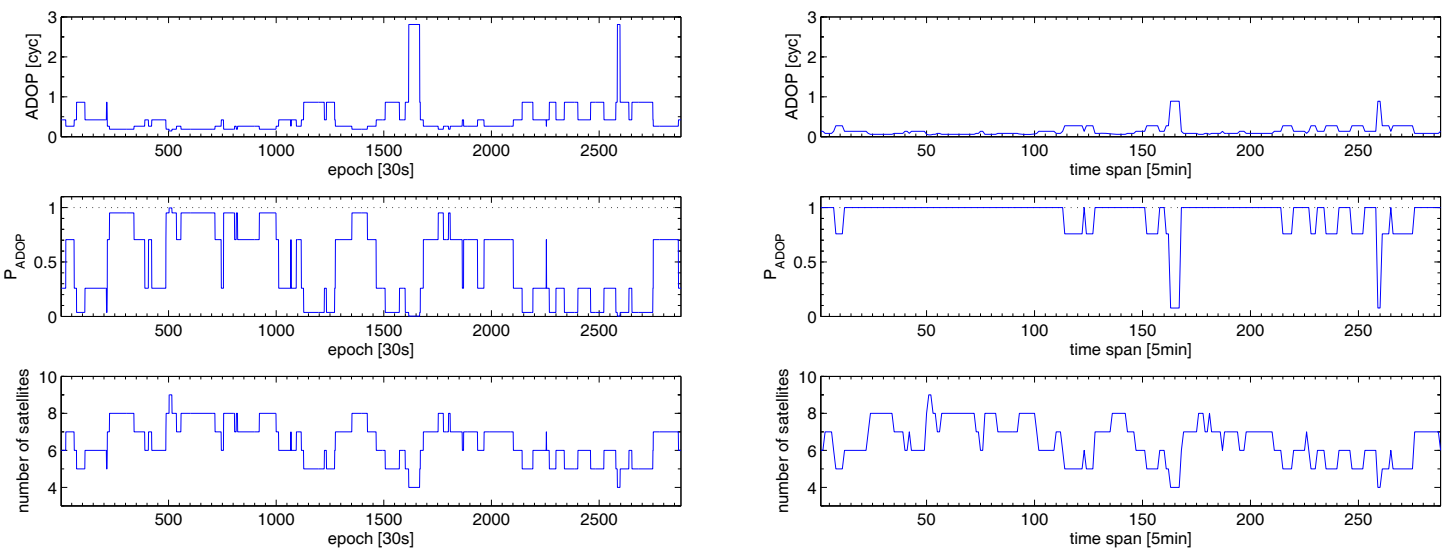

Figure 2: $A D O P, P_{A D O P}$ and number of satellites vs. observation epochs for a singlefrequency ionosphere-fixed scenario. The left 3 graphs are based on single-epochs (instantaneous), while the right 3 graphs are based on 5-minute time spans.

Increasing the time span by enlarging the data sampling interval $T$ (and keeping the number of samples constant) will lead to the following. Suppose we increase the sampling interval with a factor $f$, then the temporal correlation between the observations will become $\exp \left\{-\frac{f T}{\tau}\right\}=\left(\exp \left\{-\frac{T}{\tau}\right\}\right)^{f}$. Thus $\beta$ in the correlation matrix $R_{k}$ should be replaced by $\beta^{f}$, and this results in the following relation between the ADOP based on the original sampling interval $T$ and the ADOP based on the enlarged interval $f T$ :

$$
A D O P^{S T}(f T)=\sqrt{\frac{k-(k-2) \beta}{1+\beta} \frac{1+\beta^{f}}{k-(k-2) \beta^{f}}} A D O P^{S T}(T)
$$


Note that in both absence of time correlation $(\beta=0)$ as well as full presence of time correlation $(\beta=1)$ the multiplication factor in Eq. (17) reduces to 1, since enlarging the sampling interval does not make sense. In presence of time correlation enlarging the sampling interval is beneficial for ADOP. For example, if $\beta=0.75$ and $k=10$, enlarging of the sampling interval with a factor $f=2$ the ADOP becomes a factor 0.81 of the original ADOP.

\subsubsection{Effect for the long-time geometry-based model}

We will now look at the effect of increasing the time span in case of the long-time geometrybased model. For this model an increase of the observation time span impacts on ADOP not only through the $f_{2}$ factor, but also through $f_{5}$, because of the dependence of this factor on the baseline gain numbers. In Teunissen (1997b) it was proved that these gain numbers (minus 1$)$ are inversely proportional to the squared time span, i.e. $\gamma_{i}-1 \sim$ $1 /[(k-1) T]^{2}$.

Hence, when increasing the time span with $z$ epochs (keeping the sampling interval constant), the gain numbers at epoch $k+z$, denoted as $\gamma_{i}(k+z)$ may be related to the gain numbers at epoch $k$, denoted as $\gamma_{i}(k)$, as follows:

$$
\gamma_{i}(k+z) \simeq\left[\frac{k-1}{k+z-1}\right]^{2} \gamma_{i}(k), \text { for } i=1, \ldots, v
$$

Unfortunately, the impact of this scaling of the gain numbers on ADOP factor $f_{5}$ cannot be easily investigated, since the gain numbers appear in both the denominator and numerator of the ratio in $f_{5}$. There is however an exception. In absence of code data namely, we have $\delta=0$ and factor $f_{5}$ reduces to $\left[\prod_{i=1}^{v} \gamma_{i}\right]^{\frac{1}{2 j(m-1)}}$. Consequently, the scaling of the gain numbers in Eq. (18) is simply propagated as a factor $\left[\frac{k-1}{k+z-1}\right]^{2 v}$ in $f_{5}$. And thus, the phase-only long-time ADOP at epoch $k+z$ can be approximated as the following function of the ADOP based on $k$ epochs:

$$
A D O P_{\phi}^{L T}(k+z) \simeq \sqrt{\frac{k}{k+z}}\left[\frac{k-1}{k+z-1}\right]^{\frac{v}{j(m-1)}} A D O P_{\phi}^{L T}(k)
$$

For the example with $k=2$ and $z=20$, the ADOP corresponding to 22 epochs is only 0.07 times the two-epoch ADOP in case of four satellites, two frequencies and $v=3$. In case of eight satellites, this factor is 0.16 .

When the observation time span is increased by enlarging the data sampling interval with a factor $f$ (keeping the number of samples constant), then we have the following relation for the gain numbers, since they are inversely proportional to the sampling interval $T$ :

$$
\gamma_{i}(f T) \simeq \frac{1}{f^{2}} \gamma_{i}(T), \quad \text { for } i=1, \ldots, v
$$

This enlargement of the sampling interval results in the following phase-only ADOP:

$$
A D O P_{\phi}^{L T}(f T) \simeq f^{-\frac{v}{j(m-1)}} A D O P_{\phi}^{L T}(T)
$$

For example, in case of four satellites, two frequencies and $v=3$, enlarging the sampling interval with a factor $f=10$ implies that the ADOP becomes a factor 0.32 of the original ADOP. 


\subsection{Effect of ionospheric weighting}

If the length of a GNSS baseline is sufficiently short, the differential ionospheric delays may usually be neglected. For longer baselines this is not allowed, since they may hamper ambiguity resolution and bias the final coordinates. In that case one may parameterize the ionospheric delays as unknown parameters (which is more or less equivalent by taking the ionosphere-free combination of GPS dual-frequency observables), though ambiguity resolution will become problematic in case of short observation time spans. A solution to this might be the weighting of ionospheric delays in the single baseline model through the modeling of ionospheric pseudo-observables. For example, in case of network RTK (e.g. Wanninger, 1995) ionospheric corrections as interpolated from the ionospheric delays estimated at the network stations can be chosen as sample values of the pseudo-observables for the baseline to be determined. The standard deviation or weight of these pseudoobservables can then be chosen as function of the distance of the user's receiver to the network's master reference station, see e.g. Odijk (2000) and Wielgosz et al. (2005). This way of weighting the ionospheric delays is more or less comparable to the Quasi Ionosphere Free strategy for ambiguity resolution as implemented in the Bernese GPS Software (Dach et al., 2007). In this subsection we will investigate the effect of this ionospheric weighting on ADOP. It will be clear that for all single-baseline models this ionosphere-weighted ADOP interpolates between its ionosphere-fixed $\left(c_{2}^{2}=0\right.$; the approach for sufficiently short baselines) and ionosphere-float $\left(c_{\imath}^{2}=\infty\right.$; the approach for long baselines) counterparts:

$$
A D O P\left(c_{\imath}^{2}=0\right)<A D O P\left(0<c_{\imath}^{2}<\infty\right)<A D O P\left(c_{\imath}^{2}=\infty\right)
$$

This is of course not surprising, since the less precise the ionospheric constraints, the weaker the model and the larger ADOP becomes. In practice this means that when the ionospheric variance factor is chosen as function of the baseline length, the ADOP becomes larger the longer the baseline.

To quantify the impact of the ionospheric weighting on ADOP we need to evaluate factors $f_{4}$ and $f_{5}$. To be more specific: scalars $\iota$ and $\delta$, see Eqs. (5) and (7), need to be investigated. Since the expressions for $\iota$ and $\delta$ are quite complex we will first simplify them by relaxing the stochastic model assumptions somewhat.

\subsubsection{Simplified expressions for $\iota$ and $\delta$}

We first assume that correlation between the phase observables of different frequencies and the code observables of different frequencies is absent. In that case both cofactor matrices $C_{\phi}$ and $C_{p}$ become diagonal matrices. In addition, when all phase observables and all code observables have equal variances, we may derive expressions for $\iota$ and $\delta$ in terms of observable weights. In Eqs. (6) and (8) we insert the following observable weight matrices:

$$
C_{\phi}^{-1}=w_{\phi} I_{j}, \quad C_{p}^{-1}=w_{p} I_{j}, \quad c_{\imath}^{-2}=w_{\imath}
$$

where $w_{\phi}=1 / c_{\phi}^{2}$ denotes the weight of all phase observables, $w_{p}=1 / c_{p}^{2}$ the weight of all code observables and $w_{\imath}=1 / c_{\imath}^{2}$ the weight of the ionosphere in the model (note: ionosphere-fixed: $w_{\imath}=\infty$; ionosphere-float: $w_{\imath}=0$ ). After some elaborations we find the following simplified expressions for $\iota$ and $\delta$ in terms of these observable weights:

$$
\iota=\frac{1}{\sum_{i=1}^{j} \mu_{i}^{2}} \frac{w_{\imath}}{w_{\phi}}+\frac{w_{p}}{w_{\phi}}
$$


and

$$
\delta=\frac{\frac{w_{p}}{w_{\phi}}\left[\frac{w_{p}}{w_{\phi}} \sum_{i=1}^{j}\left(\mu_{i}-\bar{\mu}\right)^{2}+\frac{w_{2}}{w_{\phi}}\right]\left[\left(1+\frac{w_{p}}{w_{\phi}}\right) \sum_{i=1}^{j} \mu_{i}^{2}+\frac{w_{2}}{w_{\phi}}\right]}{\frac{1}{j} \sum_{l=1}^{j}\left(\left[\sum_{i=1}^{j} \mu_{i}\left(\mu_{l}+\mu_{i}\right)\right] \frac{w_{p}}{w_{\phi}}+\frac{w_{2}}{w_{\phi}}\right)^{2}+\sum_{i=1}^{j}\left(\mu_{i}-\bar{\mu}\right)^{2}\left(\frac{w_{p}}{w_{\phi}} \sum_{i=1}^{j} \mu_{i}^{2}+\frac{w_{2}}{w_{\phi}}\right)}
$$

Note that in the second expression we have made use of the equality $\sum_{i=1}^{j}\left(\mu_{i}-\bar{\mu}\right)^{2}=$ $\sum_{i=1}^{j} \mu_{i}^{2}-j \bar{\mu}^{2}$, where $\bar{\mu}=\frac{1}{j} \sum_{i=1}^{j} \mu_{i}$ is the arithmetic mean of the squared wavelength ratios. Eqs. (24) and (25) show that both factors $\iota$ and $\delta$ are governed by ratios of the observable weights, rather than their individual weights. These ratios are the code-phase weight ratio $w_{p} / w_{\phi}$ and the ionosphere-phase weight ratio $w_{\imath} / w_{\phi}$.

\subsubsection{Effect of ionospheric weighting in case of GPS}

Factor $f_{4}$ is a function of $\iota$ and from Eq. (24) it can be directly inferred that factor $\iota$ is proportional with $w_{\imath}$. This implies that the larger the ionospheric weight, the smaller $1+\frac{1}{\iota}$, which is beneficial since the ADOP becomes smaller. With an infinitely large ionospheric weight $\left(w_{\imath}=\infty\right)$, i.e. when the ionospheric delays are hard constrained or zero, $\iota$ becomes infinitely large, but the ADOP still exists since $1+\frac{1}{\iota}$ reduces to 1 . In that case the ADOP of the ionosphere-fixed model is obtained. With a zero ionospheric weight $\left(w_{\imath}=0\right)$ on the other hand, i.e. when the ionospheric delays are not constrained at all, the factor $\iota$ reduces to the ratio of the weights of the code and phase data, $w_{p} / w_{\phi}$. Usually, this ratio is in the order of $10^{-4}$. This small ratio has a very deteriorating effect on the (ionosphere-float) ADOP, since its reciprocal value, which is a large $10^{4}$, remains in the ADOP expression. Figure 3 shows factor $1+\frac{1}{\iota}$ for the three GPS frequencies (see Table 1) as function of $w_{\imath} / w_{\phi}$.

Table 1: Frequencies and wavelengths of (modernized) GPS

\begin{tabular}{lll}
\hline signal & frequency $(\mathrm{MHz})$ & wavelength $(\mathrm{cm})$ \\
\hline L1 & $154 \times 10.23=1575.42$ & 19.03 \\
L2 & $120 \times 10.23=1227.60$ & 24.42 \\
L5 & $115 \times 10.23=1176.45$ & 25.48 \\
\hline
\end{tabular}

As mentioned, the a priori ionospheric weighting also affects ADOP-factor $f_{5}$. This factor is a function of $\delta$ and in the ionosphere-fixed case $\left(w_{\imath}=\infty\right)$, Eq. (25) simply reduces to the code-phase weight ratio:

$$
\delta\left(w_{\imath}=\infty\right)=\frac{w_{p}}{w_{\phi}}
$$

In the ionosphere-float case $\left(w_{\imath}=0\right)$, Eq. $(25)$ reduces to:

$$
\begin{aligned}
\delta\left(w_{\imath}=0\right) & =\frac{\left(\frac{w_{p}}{w_{\phi}}\right)^{2}\left(1+\frac{w_{p}}{w_{\phi}}\right)\left[\sum_{i=1}^{j}\left(\mu_{i}-\bar{\mu}\right)^{2}\right]\left[\sum_{i=1}^{j} \mu_{i}^{2}\right]}{\left(\frac{w_{p}}{w_{\phi}}\right)^{2} \frac{1}{j} \sum_{l=1}^{j}\left[\sum_{i=1}^{j} \mu_{i}\left(\mu_{l}+\mu_{i}\right)\right]^{2}+\frac{w_{p}}{w_{\phi}}\left[\sum_{i=1}^{j}\left(\mu_{i}-\bar{\mu}\right)^{2}\right]\left[\sum_{i=1}^{j} \mu_{i}^{2}\right]} \\
& =\frac{\left(1+\frac{w_{p}}{w_{\phi}}\right)\left[\sum_{i=1}^{j}\left(\mu_{i}-\bar{\mu}\right)^{2}\right]\left[\sum_{i=1}^{j} \mu_{i}^{2}\right]}{\frac{1}{j} \sum_{l=1}^{j}\left[\sum_{i=1}^{j} \mu_{i}\left(\mu_{l}+\mu_{i}\right)\right]^{2}+\frac{w_{\phi}}{w_{p}}\left[\sum_{i=1}^{j}\left(\mu_{i}-\bar{\mu}\right)^{2}\right]\left[\sum_{i=1}^{j} \mu_{i}^{2}\right]}
\end{aligned}
$$

Since $w_{p} / w_{\phi} \ll 1$, it is allowed to approximate the numerator of the lower ratio in Eq. (27) neglecting this very small code-phase weight ratio. The same reasoning applies to the denominator of this ratio: due to multiplication with the phase-code weight ratio 


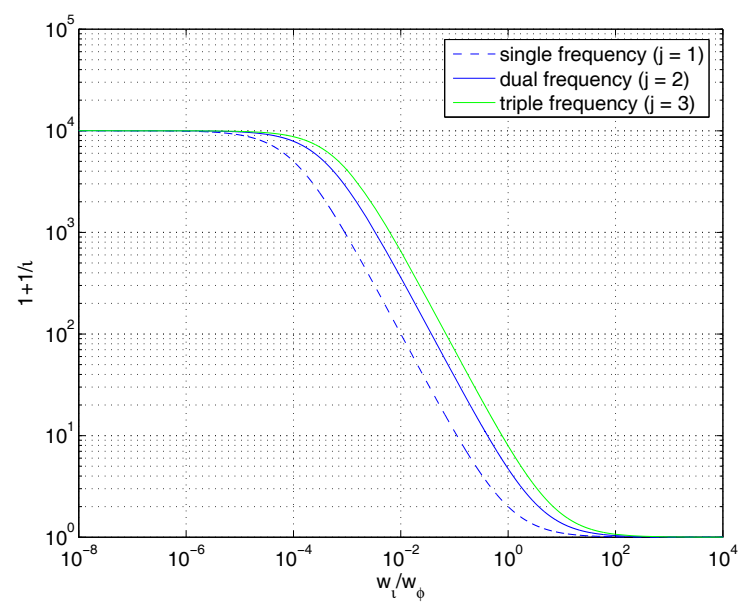

Figure 3: Factor $1+\frac{1}{\iota}$ as function of $w_{\imath} / w_{\phi}$, where it is assumed that $w_{\phi} / w_{p}=10^{4}$.

$w_{\phi} / w_{p}$, which is much larger than 1 , the second term (at the right side of the plus sign) dominates over the first term in the denominator. Hence, it is allowed to simplify the ionosphere-float $\delta$ as:

$$
\delta\left(w_{\imath}=0\right) \simeq \frac{\left[\sum_{i=1}^{j}\left(\mu_{i}-\bar{\mu}\right)^{2}\right]\left[\sum_{i=1}^{j} \mu_{i}^{2}\right]}{\frac{w_{\phi}}{w_{p}}\left[\sum_{i=1}^{j}\left(\mu_{i}-\bar{\mu}\right)^{2}\right]\left[\sum_{i=1}^{j} \mu_{i}^{2}\right]}=\frac{w_{p}}{w_{\phi}}, \quad j \geq 2
$$

Thus, in both ionosphere-fixed and ionosphere-float cases factor $\delta$ reduces to the small code-phase weight ratio $w_{p} / w_{\phi}$. Note that in the single-frequency case $(j=1)$ this does not apply, since then $\bar{\mu}=\mu_{1}$ and consequently $\sum_{i=1}^{j}\left(\mu_{i}-\bar{\mu}\right)^{2}=0$. In that case the numerator of Eq. (27) becomes zero and thus $\delta\left(w_{\imath}=0\right)=0$. This is of course due to the fact that the geometry-free and short-time ionosphere-float models are not solvable using single-frequency (phase and code) data only. The long-time ionosphere-float model can however be solved, since for this model factor $f_{5}$ does not approach infinity when $\delta\left(w_{\imath}=0\right)=0$, see Eq. (7). Table 2 summarizes the (approximated) factors $\iota$ and $\delta$ in the ionosphere-fixed and -float cases.

Table 2: Factors $\iota$ and $\delta$ for the ionosphere-fixed and -float cases.

\begin{tabular}{lllll}
\hline & \multicolumn{3}{c}{ ionosphere-fixed $\left(w_{\imath}=\infty\right)$} & \multicolumn{2}{c}{ ionosphere-float $\left(w_{\imath}=0\right)$} \\
& $\iota$ & $\delta$ & $\iota$ & $\delta$ \\
\hline single-frequency & $\infty$ & $\frac{w_{p}}{w_{\phi}}$ & $\frac{w_{p}}{w_{\phi}}$ & 0 \\
multi-frequency & $\infty$ & $\frac{w_{p}}{w_{\phi}}$ & $\frac{w_{p}}{w_{\phi}}$ & $\simeq \frac{w_{p}}{w_{\phi}}$ \\
\hline
\end{tabular}

If the ionospheric weight takes on a value in between the extremes, i.e. if $0<w_{\imath}<\infty$, then it is not quite clear what the effect will be on ADOP factor $f_{5}$, since $w_{\imath} / w_{\phi}$ appears in both the numerator and denominator of the ratio in Eq. (25). Therefore in Fig. 4 factor $1+\frac{1}{\delta}$ is plotted as function of $w_{\imath} / w_{\phi}$ for the single-, dual- and triple-frequency GPS cases. The figure shows that this factor is not insensitive to the ionospheric weighting, despite that in the ionosphere-fixed and -float cases the factor is approximately equal 
to $\left(1+w_{\phi} / w_{p}\right)^{\frac{1}{2 j}}$. In the dual- and triple-frequency cases a minimum value of factor $1+\frac{1}{\delta}$ is reached at $w_{\imath} / w_{\phi} \simeq 0.01$. In the single-frequency case a minimum is reached at $w_{\imath} / w_{\phi} \simeq 0.0002$, while the factor grows to infinity the smaller $w_{\imath} / w_{\phi}$. Despite this behavior, it can be proved that as consequence of the multiplication of factor $f_{5}$ with $f_{4}$ it is suppressed in ADOP. Hence, ADOP is a monotone decreasing function of $w_{\imath} / w_{\phi}$.

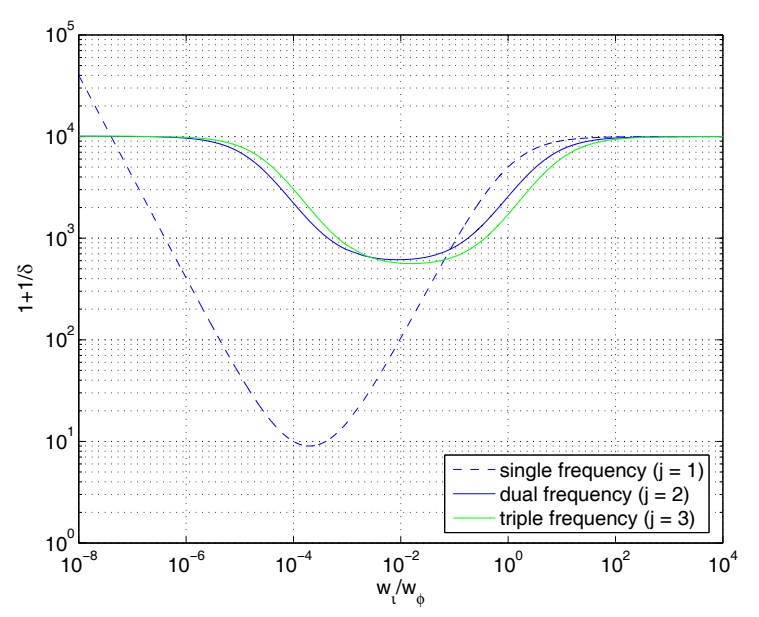

Figure 4: Factor $1+\frac{1}{\delta}$ as function of $w_{\imath} / w_{\phi}$, where it is assumed that $w_{\phi} / w_{p}=10^{4}$.

We also have to look at the cases that either $\iota$ and/or $\delta$ become zero, since in those cases $\mathrm{ADOP}$ fails to exist. In general, $\delta$ becomes zero when no code data $\left(w_{p}=0\right)$ are included. In that case the geometry-free and short-time ADOPs fail to exist, since these models are not solvable in absence of code data. If $\delta=0$ the long-time ADOP can still be computed, since in that case factor $f_{5}$ reduces to $\left[\prod_{i=1}^{v} \gamma_{i}\right]^{\frac{1}{2(m-1) j}}$. However, at least two observation epochs are needed in the phase-only case (since the single-epoch gain numbers approach infinity). In absence of code data also the geometry-fixed ADOP exists. The phase-only long-time and geometry-fixed ADOPs can however not be computed in ionosphere-float mode, since then $\imath=0$ (see Eq. (24)) and factor $f_{4}$ approaches towards infinity. Table 3 summarizes the versions of the ionosphere-weighted GNSS model (fixed/weighted/float) that can be solved and which cannot.

Table 3: Versions of the ionosphere-weighted model that can be solved $(\mathrm{Y})$ or not $(\mathrm{N})$.

\begin{tabular}{|c|c|c|c|c|c|c|}
\hline \multirow[b]{2}{*}{ observables } & \multicolumn{3}{|c|}{ GFr, MR-ST, SR-ST } & \multicolumn{2}{|c|}{ GFi, SR-LT } & \multirow[b]{2}{*}{ float } \\
\hline & fixed & weighted & float & fixed & weighted & \\
\hline$\phi_{1}$ & $\mathrm{~N}$ & $\mathrm{~N}$ & $\mathrm{~N}$ & $\mathrm{Y}$ & Y & $\mathrm{N}$ \\
\hline$\phi_{1}, \ldots, \phi_{j}$ & $\mathrm{~N}$ & $\mathrm{~N}$ & $\mathrm{~N}$ & Y & Y & $\mathrm{N}$ \\
\hline$\phi_{1}, p_{1}$ & $\mathrm{Y}$ & $\mathrm{Y}$ & $\mathrm{N}$ & Y & Y & Y \\
\hline $\begin{array}{l}\phi_{1}, \ldots, \phi_{j} \\
p_{1}, \ldots, p_{j} \\
\end{array}$ & $\mathrm{Y}$ & $\mathrm{Y}$ & $\mathrm{Y}$ & $\mathrm{Y}$ & $\mathrm{Y}$ & $\mathrm{Y}$ \\
\hline
\end{tabular}

The effect of the weighting of the ionospheric delays on ADOP can now be quantified by expressing the ionosphere-weighted ADOP as function of the ionosphere-fixed ADOP. 
Since this is not easy for the long-time model because of the presence of the gain numbers in factor $f_{5}$, we give lower and upper bounds. For the lower bound it is assumed that the gain numbers are infinitely large and for the upper bound the gain numbers are equal to one. In that case the upper bound results correspond to the short-time models and the lower bound results to the geometry-fixed model. The results for the long-time model will then interpolate between these bounds. Thus, using the property that $w_{\phi} / w_{p} \gg 1$, we find:

$$
\begin{aligned}
& A D O P\left(0<w_{\imath}<\infty\right) \simeq\left[1+\frac{1}{l}\right]^{\frac{1}{2 j}} \times \\
& \begin{cases}{\left[1+\frac{1}{\delta}\right]^{\frac{v}{2 j(m-1)}}\left(\frac{w_{\phi}}{w_{p}}\right)^{-\frac{v_{v}}{2 j(m-1)}} A D O P\left(w_{\imath}=\infty\right),} & \text { for } \gamma_{i}=\infty \\
A D O P\left(w_{\imath}=\infty\right), & \text { for } \gamma_{i}=1\end{cases}
\end{aligned}
$$

Here the actual values of $1+\frac{1}{\iota}$ and $1+\frac{1}{\delta}$ depend on the ratio of ionospheric and phase weights. These values can be extracted from Figs. 3 and 4 . In case the ionospheric weight is set to zero, from these figures it follows for $j \geq 2$ that $1+\frac{1}{\iota} \simeq 1+\frac{1}{\delta} \simeq w_{\phi} / w_{p}$, such that the ionosphere-float ADOP can be easily connected to its ionosphere-fixed counterpart:

$$
\operatorname{ADOP}\left(w_{\imath}=0\right) \simeq\left(\frac{w_{\phi}}{w_{p}}\right)^{\frac{1}{2 j}} \operatorname{ADOP}\left(w_{\imath}=\infty\right), \quad j \geq 2
$$

\subsection{Effect of the number of frequencies}

From GPS practice it is known that ambiguity resolution using data of two frequencies performs better than using single-frequency data. From the previous subsection it could already be inferred from factors $f_{4}$ and $f_{5}$ that the ADOP becomes indeed smaller when adding a frequency. In this subsection we will quantify this improvement.

The number of frequencies $j$ not only impacts on ADOP through factors $f_{4}$ and $f_{5}$, but also through factor $f_{3}$. However, factor $f_{3}$ turns out to be rather insensitive to the number of frequencies. In the numerator of this factor the number of frequencies appears in the power of the determinant of the phase cofactor matrix $C_{\phi}$. Assuming that the precision of the phase observations at different frequencies is the same (a reasonable assumption in GPS practice), i.e. $C_{\phi}=w_{\phi}^{-1} I_{j}$. Consequently, the numerator of $f_{3}$ reduces to $\left|C_{\phi}\right|^{\frac{1}{2 j}}=\frac{1}{\sqrt{w_{\phi}}}$, thus independent of the number of frequencies. In the denominator of factor $f_{3}$ the geometric mean of the wavelengths $(\breve{\lambda})$ appears and this mean depends on the number as well as the size of the wavelengths. From this follows that ADOP benefits when the data are transmitted at a longer wavelength (or: lower frequency), since the geometric mean increases. However, since the GNSS frequencies are reasonably close together, this mean is rather insensitive to the number of frequencies. For example, if we have $j$ frequencies available and we add a frequency, than it can be proved that factor $f_{3}$ is multiplied by $\prod_{i=1}^{j}\left(\frac{\lambda_{i}}{\lambda_{j+1}}\right)^{\frac{1}{j(j+1)}}$ when going from $j$ to $j+1$ frequencies. In case of GPS, when adding a second frequency, this factor is 0.88 . When going from two frequencies to a foreseen triple-frequency case this factor increases to 0.95 , even closer to 1.

Considering the two other factors, $f_{4}$ and $f_{5}$, it can immediately be inferred that increasing the number of frequencies $j$ is beneficial, because the power $\frac{1}{j}$ reduces when $j$ increases. However, the amount of improvement depends on the way the ionosphere is weighted and on the treatment of the geometry in the models. Concerning the weighting of the ionosphere, for the sake of simplicity we distinguish between the improvement in 
case of the ionosphere-fixed model on the one hand and in case of the ionosphere-float model on the other hand. From Section 4.2 we know that the improvement in case of a true ionosphere weighting will interpolate between these two extremes. Concerning the geometry, a distinction is made between the case in which the gain numbers are infinitely large on the one hand and the case in which the gain numbers are equal to one on the other hand. The results for all other gain numbers will then interpolate between these two extreme cases.

In the ionosphere-fixed case, the ADOP based on $j+1$ frequencies can be approximated as function of the ADOP based on $j$ frequencies, as (again using the fact that $w_{\phi} / w_{p} \gg 1$ ):

$$
\begin{aligned}
& A D O P_{j+1}\left(w_{\imath}=\infty\right) \simeq \prod_{i=1}^{j}\left(\frac{\lambda_{i}}{\lambda_{j+1}}\right)^{\frac{1}{j(j+1)}} \times \\
& \begin{cases}\left(\frac{w_{\phi}}{w_{p}}\right)^{-\frac{v}{2 j(j+1)(m-1)}} A D O P_{j}\left(w_{\imath}=\infty\right), & \text { for } \gamma_{i}=\infty \\
A D O P_{j}\left(w_{\imath}=\infty\right), & \text { for } \gamma_{i}=1\end{cases}
\end{aligned}
$$

To evaluate the ionosphere-float case, we use Eq. (30). For $j+1$ frequencies this relation reads

$$
A D O P_{j+1}\left(w_{\imath}=0\right) \simeq\left(\frac{w_{\phi}}{w_{p}}\right)^{\frac{1}{2(j+1)}} A D O P_{j+1}\left(w_{\imath}=\infty\right)
$$

For the ADOP based on the right side of this relation we may now insert the approximation in Eq. (31). We then get an approximation for the ionosphere-float ADOP based on $j+1$ frequencies as function of the ionosphere-fixed ADOP based on $j$ frequencies. For this ionosphere-fixed ADOP we may insert the inverse of the approximation in Eq. (30):

$$
A D O P_{j}\left(w_{\imath}=\infty\right) \simeq\left(\frac{w_{\phi}}{w_{p}}\right)^{-\frac{1}{2 j}} \operatorname{ADOP}_{j}\left(w_{\imath}=0\right), \quad j \geq 2
$$

Finally, the ionosphere-float ADOP based on $j+1$ frequencies can be approximated as the following function of its $j$-frequency counterpart (for $j \geq 2$ ):

$$
\begin{aligned}
& A D O P_{j+1}\left(w_{\imath}=0\right) \simeq \prod_{i=1}^{j}\left(\frac{\lambda_{i}}{\lambda_{j+1}}\right)^{\frac{1}{j(j+1)}} \times\left(\frac{w_{\phi}}{w_{p}}\right)^{-\frac{1}{2 j(j+1)}} \times \\
& \begin{cases}\left(\frac{w_{\phi}}{w_{p}}\right)^{-\frac{v}{2 j(j+1)(m-1)}} A D O P_{j}\left(w_{\imath}=0\right), & \text { for } \gamma_{i}=\infty \\
A D O P_{j}\left(w_{\imath}=0\right), & \text { for } \gamma_{i}=1\end{cases}
\end{aligned}
$$

Thus in the ionosphere-float case the multiplication factors when adding a frequency are the factors of the ionosphere-fixed case, but now multiplied by $\left(w_{\phi} / w_{p}\right)^{-\frac{1}{2 j(j+1)}}$.

As in Fig. 2, Fig. 5 depicts ADOPs, ADOP-based success rates and number of satellites during the day, but now for a fictitious long baseline, such that the ionospheric delays should be accounted for. In Fig. 5 the single-epoch ionosphere-float model is used for this purpose. The left graphs depict the dual-frequency GPS case, while the right three counterparts show their future triple-frequency GPS counterparts. As can be seen, in the dual-frequency case the ADOP-based ambiguity success rates are very poor (close to zero all day) because the ADOPs are very large. In the triple-frequency case there is significant improvement. The improvement factor, computed using Eq. (34), reads with $v=3$ and $j=2$ as $0.95 \cdot\left(10^{4}\right)^{-\frac{1}{2 \cdot 2 \cdot 3}} \cdot\left(10^{4}\right)^{-\frac{3}{2 \cdot 2 \cdot 3 \cdot(m-1)}}=0.95 \cdot 10^{-\frac{1}{3}} \cdot 10^{-\frac{1}{m-1}}$. For $m=6$ this yields a factor 0.28 when adding a frequency to the ionosphere-float model. From the figures 
it can be seen that the ADOPs improve significantly and also the success rates based on these ADOPs, although these success rates are not close enough to 1 to expect successful instantaneous ambiguity resolution in the future triple-frequency GPS situation.
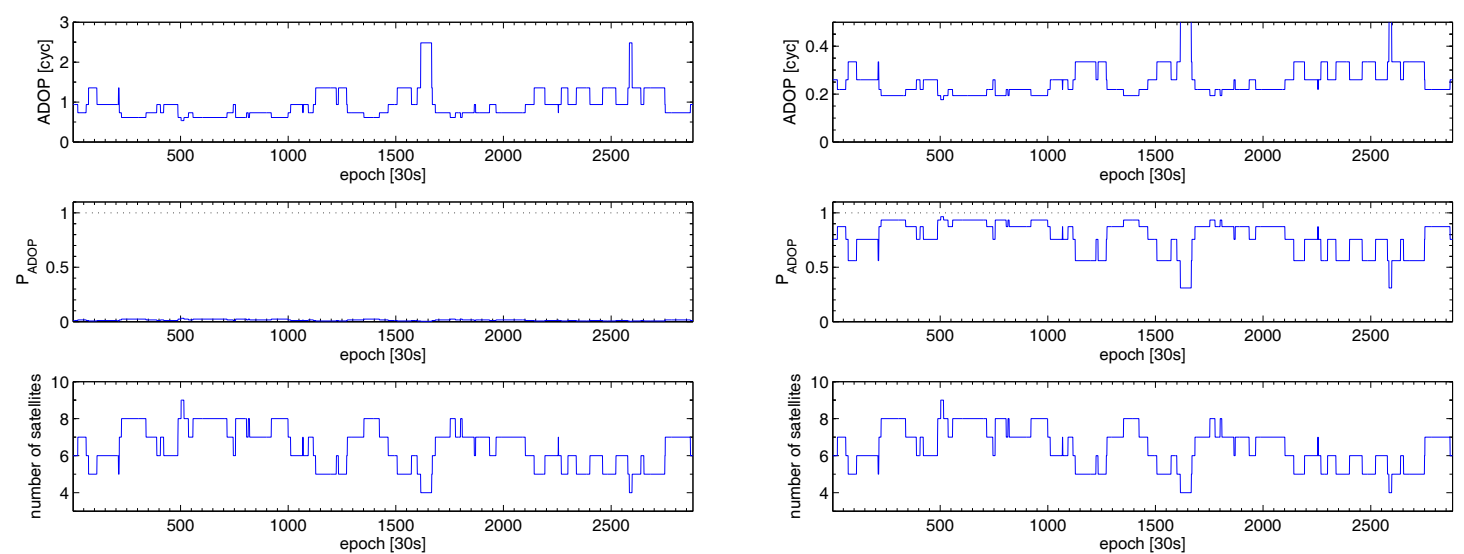

Figure 5: $A D O P, P_{A D O P}$ and number of satellites vs. observation epochs for single-epoch ionosphere-float scenario. The left 3 graphs are based on GPS dual frequency and the right 3 graphs on GPS triple frequency scenario.
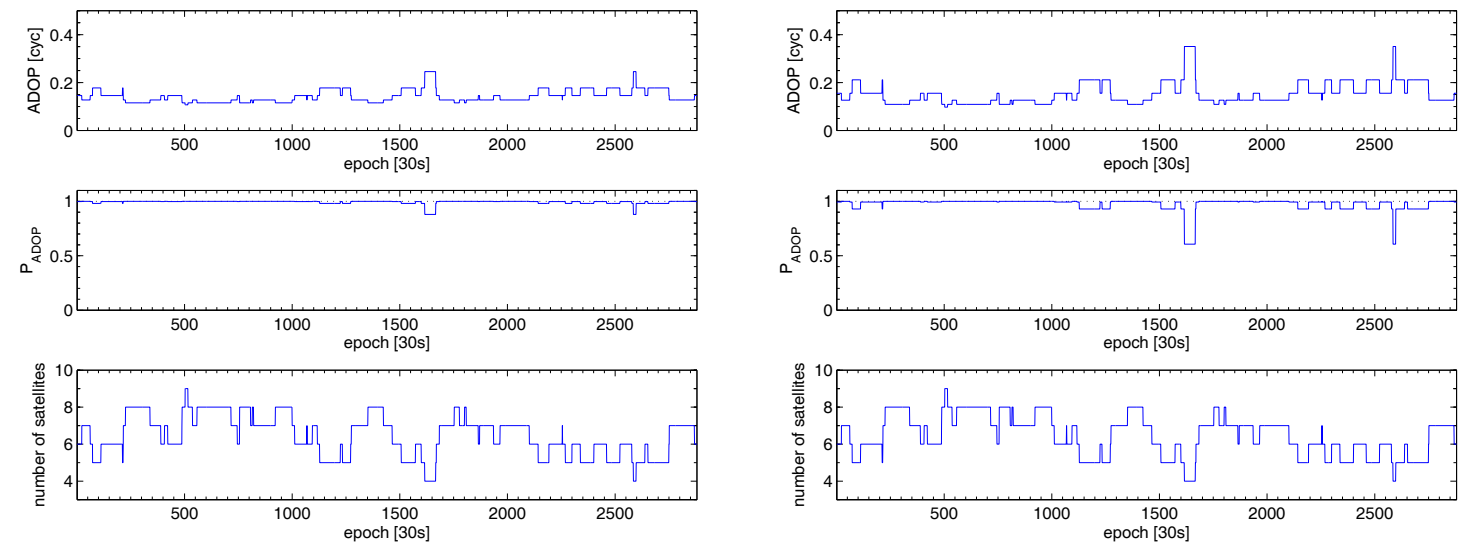

Figure 6: $A D O P, P_{A D O P}$ and number of satellites vs. observation epochs for triplefrequency ionosphere-float scenario. The left 3 graphs are based on improved code precision (standard deviation from 30 to $10 \mathrm{~cm}$ ), while the graphs on the right correspond to improved phase precision (standard deviation from 3 to $1 \mathrm{~mm}$ ). These improved cases should be compared to the three right-hand side graphs of Fig. 5.

\subsection{Effect of the code and phase weights}

Improvement of the code precision by a factor $f$, i.e. $\bar{c}_{p}=(1 / f) c_{p}$, implies that the codephase weight ratio is changed by its squared factor, i.e. $\bar{w}_{p} / w_{\phi}=f^{2} w_{p} / w_{\phi}$. This implies that $\bar{\imath}=f^{2} \iota$ and $\bar{\delta}=f^{2} \delta$, thus both factors $f_{4}$ and $f_{5}$ are affected. As in the previous subsections we will only look at the improvement in ionosphere-fixed and ionosphere-float 
ADOPs, in case the baseline gain numbers are either infinitely large or equal to 1 . First, in the ionosphere-fixed case, factor $f_{4}$ is not affected by an improved code precision since $\iota=\infty$. For factor $\bar{\delta}$ we may approximate $1+1 / \bar{\delta} \simeq\left(1 / f^{2}\right)(1+1 / \delta)$, since it holds that $1 / \delta \gg 1$ and also $1 / \bar{\delta} \gg 1$. In case $\gamma_{i}=\infty$ we may then approximate $\bar{f}_{5}=\left(1 / f^{2}\right)^{\frac{v}{2 j(m-1)}} f_{5}$. In case $\gamma_{i}=1$, factor $f_{4}=1$ and is thus not affected by an improved code precision. In the ionosphere-float case factor $f_{5}$ is affected in the same way as in the ionosphere-fixed case. Also in the ionosphere-float case factor $f_{4}$ is affected: $\bar{f}_{4}=\left(1 / f^{2}\right)^{\frac{1}{2 j}} f_{4}$. Summarizing, if the code standard deviation is improved by a factor $f$ the ADOPs are changed as follows:

$$
A D O P_{\bar{c}_{p}}\left(w_{\imath}=\infty\right) \simeq \begin{cases}\left(\frac{1}{f}\right)^{\frac{v}{j(m-1)}} A D O P_{c_{p}}\left(w_{\imath}=\infty\right), & \text { for } \gamma_{i}=\infty \\ A D O P_{c_{p}}\left(w_{\imath}=\infty\right), & \text { for } \gamma_{i}=1\end{cases}
$$

and

$$
A D O P_{\bar{c}_{p}}\left(w_{\imath}=0\right) \simeq\left(\frac{1}{f}\right)^{\frac{1}{j}} \times \begin{cases}\left(\frac{1}{f}\right)^{\frac{v}{j(m-1)}} A D O P_{c_{p}}\left(w_{\imath}=0\right), & \text { for } \gamma_{i}=\infty \\ A D O P_{c_{p}}\left(w_{\imath}=0\right), & \text { for } \gamma_{i}=1\end{cases}
$$

Figure 6 shows the ADOPs, ADOP-based success rates and number of satellites during the day for the triple-frequency ionosphere-float GPS model with improved code precision from $30 \mathrm{~cm}$ to $10 \mathrm{~cm}$, thus $f=3$. The ADOP improvement factor can then be computed as (for $v=3$ ) $\left(\frac{1}{3}\right)^{\frac{1}{3}} \cdot\left(\frac{1}{3}\right)^{\frac{1}{m-1}}$. For example, if $m=4$ this factor reads 0.48 . The beneficial effect of the more precise code data follows when evaluating the ADOP-based success rates: these are often very close to 1 . This beneficial effect is due to the fact that the ionosphere-float model heavily relies on the code data; without code data the model would not be solvable.

When the phase precision is improved (while the code precision is not changed), this affects ADOP in three factors: besides $f_{4}$ and $f_{5}$ also in $f_{3}$. It can be easily seen that when the phase precision is improved by a factor $f$, i.e. $\bar{c}_{\phi}=(1 / f) c_{\phi}$, this is proportionally propagated into $f_{3}$, since $\bar{f}_{3}=\frac{1}{f} f_{3}$. For the ADOP factors $f_{4}$ and $f_{5}$ the effect is opposite to the effect of improving the code precision, because of their dependence on the codephase weight ratio. With the improved phase precision this ratio becomes $w_{p} / \bar{w}_{\phi}=$ $\left(1 / f^{2}\right) w_{p} / w_{\phi}$. So when the phase precision is improved by a factor $f$, the ADOPs are changed as follows

$$
A D O P_{\bar{c}_{\phi}}\left(w_{\imath}=\infty\right) \simeq \frac{1}{f} \times \begin{cases}f^{\frac{v}{j(m-1)}} A D O P_{c_{\phi}}\left(w_{\imath}=\infty\right), & \text { for } \gamma_{i}=\infty \\ A D O P_{c_{\phi}}\left(w_{\imath}=\infty\right), & \text { for } \gamma_{i}=1\end{cases}
$$

and

$$
A D O P_{\bar{c}_{\phi}}\left(w_{\imath}=0\right) \simeq \frac{1}{f} \times f^{\frac{1}{j}} \times \begin{cases}f^{\frac{v}{j(m-1)}} A D O P_{c_{\phi}}\left(w_{\imath}=0\right), & \text { for } \gamma_{i}=\infty \\ A D O P_{c_{\phi}}\left(w_{\imath}=0\right), & \text { for } \gamma_{i}=1\end{cases}
$$

Figure 6 shows ADOPs, ADOP-based success rates and number of satellites during the day for the triple-frequency ionosphere-float GPS model with improved phase precision from $3 \mathrm{~mm}$ to $1 \mathrm{~mm}$, thus $f=3$. The ADOP improvement factor can then be computed as (for $v=3$ ) $3^{\frac{1}{3}} \cdot \frac{1}{3} \cdot 3^{\frac{1}{m-1}}$. For example, if $m=4$ this factor reads 0.69. The ADOP-based success rates over the day show a similar (high) level as when the code data are improved, except when few satellites are available: in that case it is more beneficial to have more precise code data then more precise phase data. 


\subsection{Effect of the number of satellites}

For all considered models the number of satellites $m$ is at least reflected in ADOP factor $f_{1}$. If $m=2$, which is the minimum number of satellites possible (GFr and GFi models), factor $f_{1}$ equals $m^{\frac{1}{2(m-1)}}=\sqrt{2}$. With an increasing number of satellites this factor approaches towards 1 , and in the limiting case of $m=\infty, m^{\frac{1}{2(m-1)}}=1$. Thus, since $m^{\frac{1}{2(m-1)}} \in[1, \sqrt{2})$, the effect of increasing the number of satellites has only a limited effect on the size of factor $f_{1}$. The same conclusion applies to the geometry-free and geometry-fixed ADOPs, since the number of satellites only appears through factor $f_{1}$. For the other models the number of satellites is also reflected in the power of factor $f_{5}$. In addition to this, in case of the long-time geometry-based ADOP the number of satellites impacts on the baseline gain numbers $\gamma_{i}$. Because of their dependence on the actual receiver-satellite geometry, the improvement in the gain numbers due to an increased number of satellites cannot be quantified analytically. However, we may evaluate the improvement in case of the two extreme values of the gain numbers. For the extreme value of $\gamma_{i}=1$ increasing the number of satellites does not have any effect, since in that case $f_{5}$ reduces to 1 . When $\gamma_{i}=\infty$ on the other hand, the ADOP corresponding to $m+q$ satellites can be approximated as the following function of the ADOP corresponding to $m$ satellites:

$$
A D O P_{m+q} \simeq \frac{(m+q)^{\frac{1}{2(m+q-1)}}}{m^{\frac{1}{2(m-1)}}}\left(\frac{w_{\phi}}{w_{p}}\right)^{-\frac{v q}{2 j(m+q-1)(m-1)}} A D O P_{m}
$$

For example, if we have a minimum of $m=4$ satellites, then a doubling of this number $(q=4)$ has as consequence that the single-frequency $(j=1)$ ADOP gets multiplied by a factor (using $v=3$ and $\left.w_{\phi} / w_{p}=10^{4}\right) \frac{8^{1 / 14}}{4^{1 / 6}}\left(10^{4}\right)^{-\frac{12}{2 \cdot 7 \cdot 3}} \simeq 0.07$, i.e. an enormous improvement! In case of dual-frequency data $(j=2)$ the multiplication factor due to a doubling of $m=8$ satellites reads (using $q=8) \frac{16^{1 / 30}}{8^{1 / 14}}\left(10^{4}\right)^{-\frac{24}{4 \cdot 15 \cdot 7}} \simeq 0.56$, i.e. still a considerable improvement.

\subsection{Effect of satellite-dependent weighting}

In GPS practice the accuracy of measurements often depends on the elevation under which the satellites are tracked. In this subsection the effect of satellite-dependent observation weighting is analyzed for the short-time geometry-based models. This effect shows up as a scale factor in factor $f_{1}$, see Eq. (2). This scale factor reads $\left[\frac{1}{m} \frac{\sum_{s=1}^{m} w_{s}}{\prod_{s=1}^{m} w_{s}}\right]^{\frac{1}{2(m-1)}}$. The elevation-dependent weights may for example be computed using an exponential function, see e.g. Euler and Goad (1991):

$$
w_{s}=1 /\left(1+\alpha \exp \left\{-\frac{\epsilon_{s}}{\epsilon_{0}}\right\}\right)^{2}
$$

Here $\epsilon_{s}$ denotes the elevation of satellite $s, \epsilon_{0}$ some reference elevation (not necessarily the cut-off elevation) and $\alpha \geq 0$ a constant. Using this function, the weight for a satellite close to the horizon will be about $1 /(1+\alpha)^{2}$, while the weight for a satellite close to the zenith will be close to 1 (when $\epsilon_{0}$ is for example set to $15 \mathrm{deg}$ ). Another elevation-dependent weighting function is simply the square of the sine of the elevation, see e.g. Vermeer (1997):

$$
w_{s}=\sin ^{2} \epsilon_{s}
$$

To investigate the limits of the scale factor in $f_{1}$ due to satellite-dependent weighting we assume that we only have satellites close to the horizon. Using the exponential function 
in Eq. (40), the weight of all satellites are then equal to $1 /(1+\alpha)^{2}$. The scale factor for ADOP then easily follows as $\left[\frac{1}{m} \frac{\sum_{s=1}^{m} w_{s}}{\prod_{s=1}^{s} w_{s}}\right]^{\frac{1}{2(m-1)}}=1+\alpha$. In general, when using a satellite weighting conform Eq. (40), the deterioration of ADOP is at most a factor $1+\alpha$ compared to its value in absence of satellite-dependent weighting.

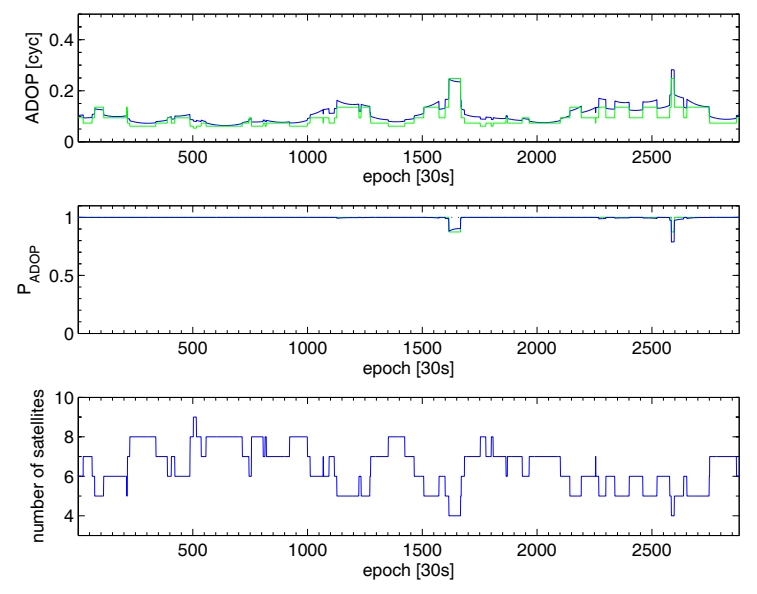

Figure 7: $A D O P, P_{A D O P}$ and number of satellites vs. observation epochs for single-epoch ionosphere-fixed scenario. The full line represents the results in presence of satellitedependent weighting, while the dotted line shows the results in absence of it.

Fig. 7 shows for the ADOPs, ADOP-based success rates and number of satellites vs. observation epoch for the 2880 single epoch time spans during our selected day. Remind that the cut-off elevation of this data set is $15 \mathrm{deg}$. The ADOPs and success rates have been computed using the dual-frequency ionosphere-fixed model. Satellitedependent weights were computed according to Eq. (40) with $\alpha=8$ and $\epsilon_{0}=15 \mathrm{deg}$ (in this case equal to the cut-off elevation). Note that using these two values the elevationdependent weights according to Eq. (40) become approximately equal to the squared sines of the elevations, see Eq. (41). For the sake of comparison the figure also shows the results in absence of satellite-dependent weighting. In order to make a fair comparison in the latter case it has been assumed that the variance factors of the phase and code data in presence of satellite-dependent weighting may be taken smaller than in absence of it. These variance factors correspond to the variances in the zenith and have been assumed at $c_{\phi}=2 \mathrm{~mm}$ and $c_{p}=20 \mathrm{~cm}$ in presence of satellite-dependent weighting, while in absence of it they are $c_{\phi}=3 \mathrm{~mm}$ and $c_{p}=30 \mathrm{~cm}$, respectively (these latter values should be considered as more or less average values valid for all satellite elevations above the cut-off angle).

As can be seen from Fig. 7, the ADOP and probability graphs do not differ much in presence or absence of satellite-dependent weighting. This is due to the fact that the effect of satellite-dependent weighting is more or less averaged out, since the weights of highelevation data become relatively large, while those of low-elevation data become relatively small compared to their counterparts in absence of satellite-dependent weighting. 


\subsection{Effect of taking linear combinations of data}

ADOP-factors $f_{1}$ up to and including $f_{5}$ have been derived in Odijk and Teunissen (2008) based on DD observables of uncombined frequencies. In GPS practice however linear combinations of dual-frequency data are frequently used. Well known are the wide-lane, narrow-lane and ionosphere-free combinations. In this subsection we will investigate the impact of such linear combinations on ADOP.

In general, define the following linear combination of the dual-frequency DD phase observations:

$$
\phi_{\alpha \beta}=\frac{\alpha \lambda_{2}}{\alpha \lambda_{2}+\beta \lambda_{1}} \phi_{1}+\frac{\beta \lambda_{1}}{\alpha \lambda_{2}+\beta \lambda_{1}} \phi_{2}
$$

where $\alpha$ and $\beta$ are two integer scalars. Note that the two coefficients, i.e. $\frac{\alpha \lambda_{2}}{\alpha \lambda_{2}+\beta \lambda_{1}}$ and $\frac{\beta \lambda_{1}}{\alpha \lambda_{2}+\beta \lambda_{1}}$ add up to 1. Applying this transformation to the model of uncombined dualfrequency phase data, we obtain the following transformed single-baseline model (assuming no correlation between the two original frequencies and equal variance factors of $\left.c_{\phi}^{2}\right)$ :

$$
\begin{aligned}
& E\left\{\phi_{\alpha \beta}\right\}=\left[\begin{array}{ll}
B & \lambda_{\alpha \beta}\left(e_{k} \otimes I_{m-1}\right)
\end{array}\right]\left[\begin{array}{c}
g \\
a_{\alpha \beta}
\end{array}\right] \\
& D\left\{\phi_{\alpha \beta}\right\}=c_{\phi_{\alpha \beta}}^{2} Q
\end{aligned}
$$

where $\lambda_{\alpha \beta}=\frac{\lambda_{1} \lambda_{2}}{\alpha \lambda_{2}+\beta \lambda_{1}}, a_{\alpha \beta}=\alpha a_{1}+\beta a_{2}$ and $c_{\phi_{\alpha \beta}}^{2}=\frac{\alpha^{2} \lambda_{2}^{2}+\beta^{2} \lambda_{1}^{2}}{\left(\alpha \lambda_{2}+\beta \lambda_{1}\right)^{2}} c_{\phi}^{2}$. Geometry matrix $B$, vector $g$ and cofactor matrix $Q$ depend on the type of single-baseline model (GFr, MRST, SR-ST, SR-LT, GFi) and are explained in Odijk and Teunissen (2008).

Because of the combined integer ambiguities that are estimable, model (43) can be considered as a single-frequency phase-only ionosphere-fixed model, with wavelength $\lambda_{\alpha \beta}$ and variance factor $c_{\phi_{\alpha \beta}}^{2}$. Consequently, the ADOP of the linearly combined ambiguities differs from the single-frequency phase-only ADOP only through factor $f_{3}$. This factor reads for model (43) as:

$$
\frac{\left(c_{\phi_{\alpha \beta}}^{2}\right)^{\frac{1}{2}}}{\lambda_{\alpha \beta}}=\frac{\sqrt{\alpha^{2} \lambda_{2}^{2}+\beta^{2} \lambda_{1}^{2}}}{\lambda_{1} \lambda_{2}} c_{\phi}
$$

while for the model of single-frequency phase data the factor reads $\frac{c_{\phi}}{\lambda_{1}}$. This implies that the ADOP of model (43) is related to the single-frequency phase-only ADOP as follows:

$$
A D O P_{\phi_{\alpha \beta}}=\sqrt{\alpha^{2}+\beta^{2} \frac{\lambda_{1}^{2}}{\lambda_{2}^{2}}} A D O P_{\phi}^{\text {single }}
$$

For GPS we may now evaluate the ADOP of for example the wide-lane linear combination of L1 and L2 phase data. In that case $\alpha=1$ and $\beta=-1$, and with a ratio of the L2 and L1 wavelengths of $\lambda_{2} / \lambda_{1}=77 / 60$ (see Table 1 ), it easily follows that that the ADOP of the wide-lane ambiguities $a_{1}-a_{2}$ is a factor 1.27 larger than the ADOP of the original L1 ambiguities. From this the conclusion follows that from a point of view of lowering ADOP, taking the wide-lane linear combination does not make any sense; the average precision of the ambiguities is even worse than in the case of the original single-frequency ambiguities. In some of the GPS literature a better performance of wide-lane ambiguity resolution is attributed to the longer wavelength of the wide-lane ambiguities; of course a wavelength of $\lambda_{\alpha \beta} \simeq 86 \mathrm{~cm}$ is longer than the GPS L1 and L2 wavelengths, but this does not guarantee a better performance, since the (measurement) noise of the wide-lane linear combination is amplified as well (with almost a factor $\sqrt{\lambda_{2}^{2}+\lambda_{1}^{2}} /\left(\lambda_{2}-\lambda_{1}\right) \simeq 6$ ). 
A linear combination that takes unmodeled ionospheric delays into account is the widely used ionosphere-free combination of GPS L1 and L2 phase data. In this case $\alpha=77$ and $\beta=-60$, since with this choice possible ionospheric delays get eliminated in the linear combination. However, despite this advantage, it seems that this combination is not suitable for fast ambiguity resolution, since from Eq. (45) it follows that the ADOP of the ionosphere-free ambiguities $77 a_{1}-60 a_{2}$ is more than a factor 90(!) larger than the ADOP of the single-frequency ambiguities.

As mentioned, by taking the linear combination in Eq. (42), the dual-frequency model of phase data reduces to a single-frequency model. In fact by doing so we loose some information that is present in the original dual-frequency observations. The information content is however preserved when we apply a one-to-one transformation to the dual-frequency phase observations: instead of forming one linear combination, two linear combinations are formed:

$$
\begin{aligned}
& \phi_{\alpha \beta}=\frac{\alpha \lambda_{2}}{\alpha \lambda_{2}+\beta \lambda_{1}} \phi_{1}+\frac{\beta \lambda_{1}}{\alpha \lambda_{2}+\beta \lambda_{1}} \phi_{2} \\
& \phi_{\gamma \delta}=\frac{\gamma \lambda_{2}}{\gamma \lambda_{2}+\delta \lambda_{1}} \phi_{1}+\frac{\delta \lambda_{1}}{\gamma \lambda_{2}+\delta \lambda_{1}} \phi_{2}
\end{aligned}
$$

with $\alpha, \beta, \gamma$ and $\delta$ scalar integers. The transformed single-baseline model becomes:

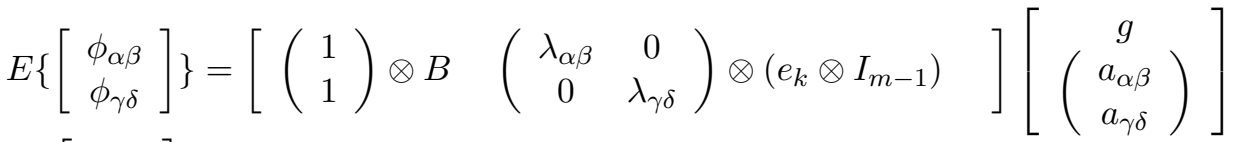

$$
\begin{aligned}
& D\left\{\left[\begin{array}{c}
\phi_{\alpha \beta} \\
\phi_{\gamma \delta}
\end{array}\right]\right\}=C_{\phi}^{L C} \otimes Q
\end{aligned}
$$

where $\lambda_{\alpha \beta}$ and $a_{\alpha \beta}$ are the same as in model (43) and where $\lambda_{\gamma \delta}=\frac{\lambda_{1} \lambda_{2}}{\gamma \lambda_{2}+\delta \lambda_{1}}$ and $a_{\gamma \delta}=$ $\gamma a_{1}+\delta a_{2}$. Moreover,

$$
C_{\phi}^{L C}=\left[\begin{array}{cc}
\frac{\alpha^{2} \lambda_{2}^{2}+\beta^{2} \lambda_{1}^{2}}{\left(\alpha \lambda_{2}+\beta \lambda_{1}\right)^{2}} & \frac{\alpha \gamma \lambda_{2}^{2}+\beta \delta \lambda_{1}^{2}}{\left(\alpha \lambda_{2}+\beta \lambda_{1}\right)\left(\gamma \lambda_{2}+\delta \lambda_{1}\right)} \\
\frac{\alpha \gamma \lambda_{2}^{2}+\beta \delta \lambda_{1}^{2}}{\left(\alpha \lambda_{2}+\beta \lambda_{1}\right)\left(\gamma \lambda_{2}+\delta \lambda_{1}\right)} & \frac{\gamma^{2} \lambda_{2}^{2}+\delta^{2} \lambda_{1}^{2}}{\left(\gamma \lambda_{2}+\delta \lambda_{1}\right)^{2}}
\end{array}\right] c_{\phi}^{2}
$$

Note from matrix $C_{\phi}^{L C}$ that the two linear combinations are correlated, whereas the original phase observables on L1 and L2 are assumed uncorrelated. Also note that model (47) is -like the original model of uncombined observations- a dual-frequency model, but with different wavelengths and phase vc-matrix. Hence, the ADOP corresponding to the linearly combined ambiguities $a_{\alpha \beta}$ and $a_{\gamma \delta}$ only differs from the ADOP of the original dual-frequency ambiguities through factor $f_{3}$. This factor is for model (47) computed as $\frac{\left|C_{\phi}^{L C}\right|^{\frac{1}{4}}}{\sqrt{\lambda_{\alpha \beta}} \sqrt{\lambda_{\gamma \delta}}}$. The determinant of the phase cofactor matrix reads $\left|C_{\phi}^{L C}\right|=$ $\frac{|\alpha \delta-\beta \gamma|^{2} \lambda_{1}^{2} \lambda_{2}^{2}}{\left(\alpha \lambda_{2}+\beta \lambda_{1}\right)^{2}\left(\gamma \lambda_{2}+\delta \lambda_{1}\right)^{2}} c_{\phi}^{4}$ and the wavelengths $\lambda_{\alpha \beta}=\frac{\lambda_{1} \lambda_{2}}{\alpha \lambda_{2}+\beta \lambda_{1}}$ and $\lambda_{\gamma \delta}=\frac{\lambda_{1} \lambda_{2}}{\gamma \lambda_{2}+\delta \lambda_{1}}$. Since factor $f_{3}$ for the uncombined dual-frequency ambiguities is $c_{\phi} / \sqrt{\lambda_{1} \lambda_{2}}$, the ADOP of model (46) is related to the dual-frequency phase-only ADOP as:

$$
A D O P_{\phi}^{L C}=\sqrt{|\alpha \delta-\beta \gamma|} A D O P_{\phi}^{\text {dual }}
$$

Note that the factor $\alpha \delta-\beta \gamma$ in the above expression corresponds to the determinant of a matrix $Z^{T}$ realizing a transformation from the original uncombined ambiguities to the linearly combined ambiguities:

$$
\left[\begin{array}{c}
a_{\alpha \beta} \\
a_{\gamma \delta}
\end{array}\right]=\underbrace{\left[\begin{array}{cc}
\alpha & \beta \\
\gamma & \delta
\end{array}\right]}_{Z^{T}}\left[\begin{array}{l}
a_{1} \\
a_{2}
\end{array}\right]
$$


From Teunissen (1995) we know that from a point of view of ambiguity resolution this transformation is only admissible when matrix $Z$ fulfils two criteria: i) it should have integer entries, and ii) the determinant of $Z$ should be equal to +1 or -1 . These criteria restrict the admissible ambiguity transformations. For example, in case of GPS it is not allowed to pair the wide-lane ambiguity to the narrow-lane ambiguity, i.e. the transformation

$$
\left[\begin{array}{c}
a_{\alpha \beta} \\
a_{\gamma \delta}
\end{array}\right]=\left[\begin{array}{cc}
1 & -1 \\
1 & 1
\end{array}\right]\left[\begin{array}{l}
a_{1} \\
a_{2}
\end{array}\right]
$$

is not admissible, since $|Z|=2 \neq \pm 1$. An admissible transformation is for example

$$
\left[\begin{array}{c}
a_{\alpha \beta} \\
a_{\gamma \delta}
\end{array}\right]=\left[\begin{array}{cc}
1 & 0 \\
1 & -1
\end{array}\right]\left[\begin{array}{l}
a_{1} \\
a_{2}
\end{array}\right]
$$

i.e. pairing the L1-ambiguity to the wide-lane ambiguity. Another admissible transformation is

$$
\left[\begin{array}{l}
a_{\alpha \beta} \\
a_{\gamma \delta}
\end{array}\right]=\left[\begin{array}{cc}
77 & -60 \\
9 & -7
\end{array}\right]\left[\begin{array}{l}
a_{1} \\
a_{2}
\end{array}\right]
$$

i.e. pairing the ionosphere-free ambiguities $77 a_{1}-60 a_{2}$ to the combination $9 a_{1}-7 a_{2}$. Thus when admissible linear combinations of the dual-frequency observables are applied, we have the important result that $|\alpha \delta-\beta \gamma|=1$ always holds. From this follows that $A D O P_{\phi}^{L C}=A D O P_{\phi}$, i.e. the ADOP remains invariant to an admissible linear combination of the phase data. A reason for applying the wide-lane in GPS practice is not that it improves the ambiguity success rate, but that it enhances the search for the integer ambiguities. Since the L1- and L2-ambiguities are highly correlated -especially using short time spans- the search for the integer solution is rather inefficient. In Teunissen (1997c) it was shown that the ambiguity transformation in Eq. (52) produces a smaller correlation coefficient between the L1 and wide-lane ambiguities than the correlation coefficient between the original L1 and L2 ambiguities, and this enhances the ambiguity search. However, the search can be enhanced much more by using the decorrelating ambiguity transformation as implemented in the $L A M B D A$ method rather than the wide-lane transformation, since it decorrelates the ambiguities much more. Note that this decorrelation is an admissible ambiguity transformation as well, see Teunissen (1995).

\section{CONCLUSIONS}

Using the closed-form expressions for ADOP as derived in Odijk and Teunissen (2008), in this contribution it was inferred how the precision of the ambiguities changes when a certain assumption in the single-baseline GNSS model is changed. In general, each of the changes impacts as (approximately) a scale factor on ADOP. In this paper these scale factors have been revealed and it could be easily seen how they depend on the other model assumptions. Table 4 summarizes these scale factors in a systematic order. In this table the effect of taking linear combinations of multiple-frequency phase data is not included, since from a point of view of improving ADOP this is not recommended.

How should Table 4 be read? For example, suppose we have single-frequency GPS L1 phase and code data from $m=4$ satellites. These data are assumed to be uncorrelated, whereas the variance factor for the phase data is $c_{\phi}=3 \mathrm{~mm}$, and the variance factor for the code data is $c_{p}=30 \mathrm{~cm}$. Suppose that we are interested in instantaneous $(k=1)$ ambiguity resolution. Let us first consider the ADOP in case the baseline and ionosphere parameters are both fixed, so the only parameters in our single-baseline model are the 
ambiguities. According to Table 4, the ADOP can be approximated (using $1 / \sqrt{w_{\phi}}=c_{\phi}$ ) as $A D O P_{\mid g}\left(w_{\imath}=\infty\right)=4^{\frac{1}{2(4-1)}} \sqrt{\frac{2}{1}} \frac{0.003}{0.1903}=0.028$ cyc. Suppose that we increase the singleepoch time span to $k=5$ epochs and that the data of these epochs are temporally correlated according to $\beta=\frac{1}{2}$, then the ADOP should be multiplied by a factor $s_{\mid g}$, which is computed (using $z=4$ ) as $\sqrt{\frac{1-(1-2) \frac{1}{2}}{1+4-(1+4-2) \frac{1}{2}}} \simeq 0.65$. Note that the code data do not contribute to the geometry-fixed, ionosphere-fixed ADOP. However, in case ionospheric delays are parameterized and these are weighted in the model, the code data do contribute. In that case the geometry-fixed ionosphere-fixed ADOP is multiplied by $\left[1+\frac{1}{\iota}\right]^{\frac{1}{2 j}}$ and this factor depends on the actual value of the ionospheric weight $w_{\imath}$ and the ratio of phase and code weights $w_{\phi} / w_{p}$. In Fig. $31+\frac{1}{\iota}$ is plotted as function of $w_{\imath} / w_{\phi}$ for $w_{\phi} / w_{p}=10^{4}$. Suppose the ionospheric variance factor is $c_{\imath}=1 \mathrm{~cm}$, then with $w_{\imath} / w_{\phi}=0.09$ from Fig. 3 follows that $1+\frac{1}{\iota} \simeq 12$ in case $j=1$. The geometry-fixed ionosphere-fixed ADOP thus gets multiplied by a factor $\sqrt{12} \simeq 3.5$ to obtain the ADOP in case of a weighted ionosphere. The ADOP becomes $0.028 \cdot 3.5 \simeq 0.097$ cyc.

Now let us consider the ADOP in case the coordinates of the second receiver of a single baseline are unknown $(v=3)$. Then, according to Table 4 , the single-epoch ADOP $\left(\gamma_{i}=\infty\right.$ for $\left.i=1, \ldots, v\right)$ can be approximated as $A D O P\left(w_{\imath}=\infty\right) \simeq A D O P_{\mid g}\left(w_{\imath}=\infty\right) \times$ $\left(w_{\phi} / w_{p}\right)^{\frac{v}{2 j(m-1)}}$. Thus, a crucial role is played by the ratio of phase and code weights, which, in our case is assumed as $w_{\phi} / w_{p}=10^{4}$. The geometry-based ionosphere-fixed ADOP is then approximated as $\operatorname{ADOP}\left(w_{\imath}=\infty\right) \simeq 0.028 \cdot\left(10^{4}\right)^{\frac{3}{2 \cdot 1 \cdot(4-1)}} \simeq 2.8$ cyc. Thus, due to the baseline estimation the ADOP increases by a factor 100! If we add a second frequency (GPS L2) to the single-frequency data, then this latter ADOP should be multiplied by two factors: i) a factor $s_{w_{l}=\infty}=\left(w_{\phi} / w_{p}\right)^{-\frac{v}{2 j(j+1)(m-1)}}$ to account for the change in the phase-code weight ratio, and ii) a factor $\prod_{i=1}^{j}\left(\lambda_{i} / \lambda_{j+1}\right)^{\frac{1}{j(j+1)}}$ to account for the change in $A D O P_{\mid g}\left(w_{\imath}=\infty\right)$. This first factor reads $\left(10^{4}\right)^{-\frac{3}{2 \cdot 1 \cdot(1+1)(4-1)}}=\left(10^{4}\right)^{-\frac{1}{4}}=0.1$, while the second factor reads $\sqrt{\frac{60}{77}} \simeq 0.88$. This yields a dual-frequency geometry-based ionospherefixed ADOP of $2.8 \cdot 0.88 \cdot 0.1 \simeq 0.25$ cyc. We will now evaluate the effects on ADOP of the ionospheric delays being weighted instead of fixed in the single-baseline model. If the ionospheric variance factor is $c_{\imath}=1 \mathrm{~cm}$, then the ionosphere-phase weight ratio is $w_{\imath} / w_{\phi}=0.09$. According to Table 4 , the geometry-based ionosphere-weighted ADOP is obtained by multiplying the ionosphere-fixed ADOP by a factor (in case of the singleepoch time spans) $\left[1+\frac{1}{\iota}\right]^{\frac{1}{2 j}} \times\left[1+\frac{1}{\delta}\right]^{\frac{v}{2 j(m-1)}} \times\left(\frac{w_{\phi}}{w_{p}}\right)^{-\frac{v}{2 j(m-1)}}$. The latter factor is 0.1 , while the first two factors are to be read from the graphs in Figs. 3 and 4 . With $w_{\imath} / w_{\phi}=0.09$ and $j=2$ from Fig. 3 follows that $1+\frac{1}{\iota} \simeq 40$, while from Fig. 4 follows that $1+\frac{1}{\delta} \simeq$ 800. Thus, the dual-frequency ionosphere-fixed ADOP should be multiplied by a factor $40^{\frac{1}{4}} \cdot 800^{\frac{3}{2 \cdot(4-1)}} \cdot 0.1 \simeq 1.34$ to obtain its ionosphere-weighted counterpart. This results in an ionosphere-weighted ADOP of 0.33 cyc. 
In Table 4 the symbols have the following meaning:

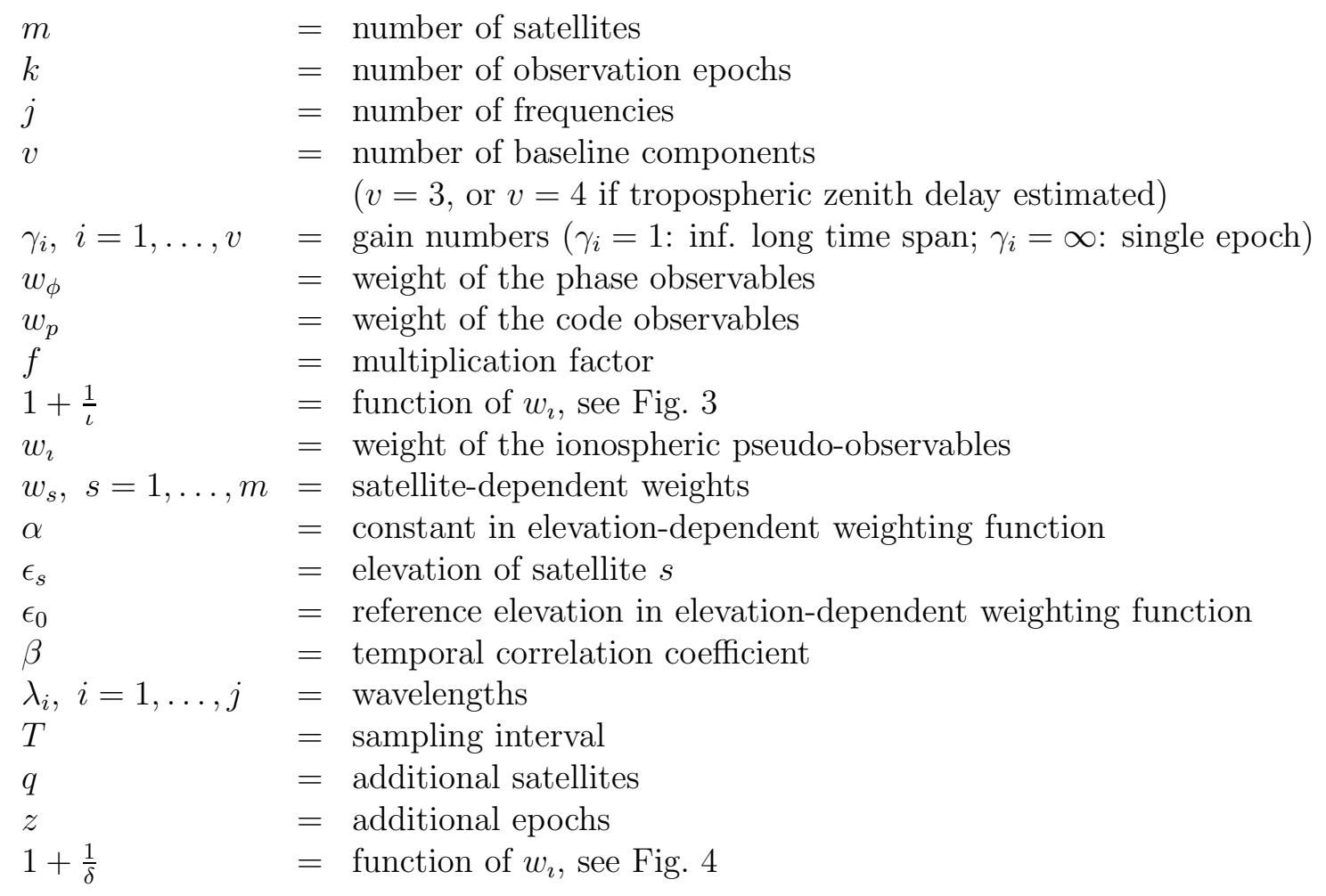

Acknowledgments. The research of Prof. Teunissen was done in the framework of his ARC International Linkage Professorial Fellowship, at the Curtin University of Technology, Perth, Australia, with Professor Will Featherstone as his host. This support is greatly acknowledged.

\section{REFERENCES}

Dach, R., U. Hugentobler, P. Fridez, and M. Meindl (eds.). Bernese GPS Software Version 5.0, User manual, Astronomical Institute, University of Bern, January 2007.

Euler, H.J., and C.C. Goad (1991). On optimal filtering of GPS dual frequency observations without using orbit information. Bulletin Géodésique 65, 130-143.

Odijk, D. (2000). Weighting ionospheric corrections to improve fast GPS positioning over medium distances. Proc. of the 13th Int. Tech. Meeting of the Satellite Division of the U.S. Institute of Navigation, ION GPS-2000, Salt Lake City, USA, September 19-22, 1113-1123.

Odijk, D., and P.J.G. Teunissen (2008). ADOP in closed form for a hierarchy of multifrequency single-baseline GNSS models. Accepted for Journal of Geodesy.

Priestley, M.B. (1981). Spectral analysis and time series. Probability and mathematical statistics, a series of monographs and textbooks, Academic Press Ltd, London/San Diego, vols. 1 and 2.

Teunissen, P.J.G. (1995). The least-squares ambiguity decorrelation adjustment: A method for fast GPS integer ambiguity estimation. Journal of Geodesy 70, 65-82. 
Teunissen, P.J.G. (1997a). A canonical theory for short GPS baselines. Part IV: Precision versus reliability. Journal of Geodesy 71, 513-525.

Teunissen, P.J.G. (1997b). A canonical theory for short GPS baselines. Part I: The baseline precision. Journal of Geodesy 71, 320-336.

Teunissen, P.J.G. (1997c). On the GPS widelane and its decorrelating property. Journal of Geodesy 71, 577-587.

Teunissen, P.J.G. (1998). The ionosphere-weighted GPS baseline precision in canonical form. Journal of Geodesy 72, 107-117.

Vermeer, M. (1997). The precision of geodetic GPS and one way of improving it. Journal of Geodesy 71, 240-245.

Wanninger, L. (1995). Improved ambiguity resolution by regional differential modelling of the ionosphere. Proc. of ION GPS-95, Palm Springs, pp. 55-62.

Wielgosz, P., I. Kashani, and D. Grejner-Brzezinska (2005). Analysis of long-range network RTK during a severe ionospheric storm. Journal of Geodesy 79, 524-531.

Received: 2007-09-04,

Reviewed: 2008-01-03, by R. Weber,

Accepted: 2008-01-12. 
Table 4: Sensitivity of ADOP to changes in the single-baseline model.

GEOMETRY-FIXED IONOSPHERE-FIXED MODEL $(m \geq 2, k \geq 1, j \geq 1)$ :

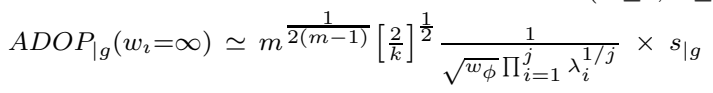

\begin{tabular}{lllll}
$\begin{array}{l}\text { model } \\
\text { change }\end{array}$ & scale factor $\left(s_{\mid g}\right)$ & lower bound scale & upper bound scale \\
\hline $\begin{array}{l}\text { satellite- } \\
\text { dependent } \\
\text { weighting }\end{array}$ & $w_{s}=\frac{1}{\left(1+\alpha \exp \left\{-\frac{\epsilon_{s}}{\epsilon_{0}}\right\}\right)^{2}}, \alpha \geq 0$ & {$\left[\frac{1}{m} \frac{\sum_{s=1}^{m} w_{s}}{\left.\prod_{s=1}^{m} w_{s}\right]^{\frac{1}{2(m-1)}}}\right.$} & 1, if $\alpha=0$ & $1+\alpha$, if $\epsilon_{s}=0$ \\
$\begin{array}{l}\text { number of } \\
\text { satellites }\end{array}$ & $m+q(q$ more satellites $)$ & $\frac{(m+q)^{\frac{1}{2(m+q-1)}}}{m^{\frac{1}{2(m-1)}}}$ & $\frac{1}{2} \sqrt{2}$, if $m=2 \wedge q=\infty$ & 1, if $m=\infty \wedge q=\infty$ \\
$\begin{array}{l}\text { time corre- } \\
\text { lation }\end{array}$ & $\operatorname{corr}\left(t_{i}, t_{j}\right)=\beta^{|j-i|}, 0 \leq \beta<1$ & $\sqrt{\frac{1+\beta}{1-(1-2 / k) \beta}}$ & 1, if $\beta=0$ & $\sqrt{k}$, if $\beta=1$ \\
time span & $k+z(z$ more epochs $)$ & $\sqrt{\frac{k-(k-2) \beta}{k+z-(k+z-2) \beta}}$ & $\sqrt{\frac{k}{k+z}}$, if $\beta=0$ & 1, if $\beta=1$ \\
& $f T($ enlarging sampling inter- & $\sqrt{\frac{k-(k-2) \beta}{1+\beta} \frac{1+\beta f}{k-(k-2) \beta f}}$ & 1, if $\beta=0$ & 1, if $\beta=1$ \\
$\begin{array}{l}\text { phase } \\
\text { weights }\end{array}$ & $f^{2} w_{\phi}(f \geq 1)$ & $\frac{1}{f}$ & 1, if $w_{\imath}=\infty$ & $\left(\frac{w_{\phi}}{w_{p}}\right)^{\frac{1}{2 j}}$, if $w_{\imath}=0$ \\
$\begin{array}{l}\text { ionospheric } \\
\text { weights }\end{array}$ & $0 \leq w_{\imath} \leq \infty, \frac{w_{p}}{w_{\phi}}=10^{-4}$ & {$\left[1+\frac{1}{l}\right]^{\frac{1}{2 j}}$} & & \\
$\begin{array}{l}\text { number of } \\
\text { frequencies }\end{array}$ & $j+1$ (one more frequency) & $\prod_{i=1}^{j}\left(\frac{\lambda_{i}}{\lambda_{j+1}}\right)^{\frac{1}{j(j+1)}}$ & &
\end{tabular}

\begin{tabular}{|c|c|c|c|}
\hline \multirow[b]{2}{*}{$\begin{array}{l}\text { model } \\
\text { change }\end{array}$} & \multicolumn{3}{|c|}{$\begin{array}{l}\text { GEOMETRY-BASED IONOSPHERE-FIXED MODEL }\left(m \geq v+1, k \geq 1, j \geq 1, \gamma_{i} \in[1, \infty)\right) \\
\qquad A D O P\left(w_{\imath}=\infty\right) \simeq A D O P_{\mid g}\left(w_{\imath}=\infty\right) \times\left[\prod_{i=1}^{v}\left(1+\frac{1-1 / \gamma_{i}}{w_{p} / w_{\phi}+1 / \gamma_{i}}\right)\right]^{\frac{1}{2 j(m-1)}} \times s_{w_{\imath}=\infty}\end{array}$} \\
\hline & & lower bound scale $\left(s_{w_{\imath}=\infty}\right)$ & upper bound scale $\left(s_{w_{\imath}}=\infty\right)$ \\
\hline $\begin{array}{l}\text { number of } \\
\text { satellites }\end{array}$ & $m+q \quad(q$ more satellites $)$ & $\left(\frac{w_{\phi}}{w_{p}}\right)^{-\frac{0 q}{2 j(m+q-1)(m-1)}}$ if $\gamma_{i}=\infty$ & $1, \quad$ if $\gamma_{i}=1$ \\
\hline \multirow[t]{2}{*}{ time span } & $k+z \quad(z$ more epochs $)$ & - & $\left(\frac{k-1}{k+z-1}\right)^{\frac{v}{j(m-1)}}$, if $\gamma_{i} \neq \infty$ \\
\hline & $\begin{array}{l}f T \text { (enlarging sampling inter- } \\
\text { val; } f \geq 1 \text { ) }\end{array}$ & - & $f^{-\frac{v}{j(m-1)}}$, if $\gamma_{i} \neq \infty$ \\
\hline $\begin{array}{l}\text { phase } \\
\text { weights }\end{array}$ & $f^{2} w_{\phi} \quad(f \geq 1)$ & $f^{\frac{v}{j(m-1)}}$ if $\gamma_{i}=\infty$ & $1, \quad$ if $\gamma_{i}=1$ \\
\hline $\begin{array}{l}\text { code } \\
\text { weights }\end{array}$ & $f^{2} w_{p} \quad(f \geq 1)$ & $\left(\frac{1}{f}\right)^{\frac{v}{j(m-1)}}$ if $\gamma_{i}=\infty$ & $1, \quad$ if $\gamma_{i}=1$ \\
\hline $\begin{array}{l}\text { ionospheric } \\
\text { weights }\end{array}$ & $0 \leq w_{\imath} \leq \infty, \frac{w_{p}}{w_{\phi}}=10^{-4}$ & {$\left[\left(1+\frac{1}{\delta}\right)\left(\frac{w_{p}}{w_{\phi}}\right)\right]^{\frac{v}{2 j(m-1)}}$ if $\gamma_{i}=\infty$} & $1, \quad$ if $\gamma_{i}=1$ \\
\hline $\begin{array}{l}\text { number of } \\
\text { frequencies }\end{array}$ & $j+1$ (one more frequency) & $\left(\frac{w_{\phi}}{w_{p}}\right)^{-\frac{v}{2 j(j+1)(m-1)}}$ if $\gamma_{i}=\infty$ & $1, \quad$ if $\gamma_{i}=1$ \\
\hline
\end{tabular}

GEOMETRY-BASED IONOSPHERE-FLOAT MODEL $\left(m \geq v+1, k \geq 1, j \geq 2, \gamma_{i} \in[1, \infty)\right)$ : $A D O P\left(w_{\imath}=0\right) \simeq A D O P\left(w_{\imath}=\infty\right) \times\left(\frac{w_{\phi}}{w_{p}}\right)^{\frac{1}{2 j}} \times s_{w_{\imath}=0}$

\begin{tabular}{lll} 
model change & & scale factor $\left(s_{w_{\imath}=0}\right)$ \\
\hline phase weights & $f^{2} w_{\phi}(f \geq 1)$ & $f^{\frac{1}{j}}$ \\
code weights & $f^{2} w_{p} \quad(f \geq 1)$ & $\left(\frac{1}{f}\right)^{\frac{1}{j}}$ \\
number of frequencies & $j+1$ (one more frequency) & $\left(\frac{w_{\phi}}{w_{p}}\right)^{-\frac{1}{2 j(j+1)}}$ \\
\hline
\end{tabular}

